\title{
Control and Optimization of Batch Chemical Processes
}

\author{
Dominique Bonvin ${ }^{1, *}$ and Grégory François ${ }^{2}$ \\ ${ }^{1}$ Laboratoire d'Automatique, Ecole Polytechnique Fédérale de Lausanne \\ EPFL - Station 9, CH-1015, Lausanne, Switzerland. \\ ${ }^{2}$ Institute for Materials and Processes, School of Engineering \\ The University of Edinburgh, Edinburgh EH9 3FB, UK. \\ * corresponding author : dominique.bonvin@epfl.ch
}

November 30, 2016

\begin{abstract}
A batch process is characterized by the repetition of time-varying operations of finite duration. Due to the repetition, there are two independent "time" variables, namely, the run time during a batch and the batch index. Accordingly, the control and optimization objectives can be defined for a given batch or over several batches. This chapter describes the various control and optimization strategies available for the operation of batch processes. These include online and run-to-run control on the one hand, and repeated numerical optimization and optimizing control on the other. Several case studies are presented to illustrate the various approaches
\end{abstract}

\section{Keywords}

Batch control, predictive control, iterative learning control, run-to-run control, batch process optimization, dynamic optimization, optimizing control, run-to-run optimization. 


\section{Introduction}

Batch processing is widely used in the manufacturing of goods and commodity products, in particular in the chemical, pharmaceutical and food industries ${ }^{1}$. Batch operation differs significantly from continuous operation. While in continuous operation the process is maintained at an economically desirable operating point, in batch operation the process state evolves from an initial to a final time. In the chemical industry for example, since the design of a continuous plant requires substantial engineering effort, continuous operation is rarely used for low-volume production. Discontinuous operations can be of the batch or semi-batch type. In batch operations, the products to be processed are loaded in a vessel and processed without material addition or removal. This operation permits more flexibility than continuous operation by allowing adjustment of the operating conditions and the final time. Additional flexibility is available in semi-batch operations, where reactants are continuously added by adjusting the feedrate profiles, or products are removed via some outflow. We use the term batch process to include semi-batch processes.

In the chemical industry, many batch processes deal with reaction and separation operations. Reactions are central to chemical processing and can be performed in an homogeneous (single phase) or heterogeneous (multi-phase) environment. Separation processes can be of very different types, such as distillation, absorption, extraction, adsorption, chromatography, crystallization, drying, filtration and centrifugation. The operation of batch processes follows recipes developed in the laboratory. A sequence of operations is performed in a pre-specified order in specialized process equipment, yielding a certain amount of product. The sequence of tasks to be carried out on each piece of equipment, such as heating, cooling, reaction, distillation, crystallization and drying, is predefined. The desired production volume is then achieved by repeating the processing steps on a predetermined schedule.

This chapter describes the various control and optimization strategies available for the operation of batch processes. Section 2 discusses the main features of batch processes, while Section 3 presents the two types of models that are available for online and run-to run operation, respectively. The rest of the chapter contains two parts, namely Part A comprising Sections 4-7 and concerned with control (online control, run- 
to-run control, batch automation, and control applications), and Part B comprising Sections 8-10 and dealing with optimization (numerical optimization, real-time optimization, and optimization applications). Finally, a summary and outlook are provided in Section 11.

\section{Features of Batch Processes}

Process engineers have developed considerable expertise in designing and operating continuous processes. On the other hand, chemists have been trained to develop new routes for synthesizing chemicals, often using a batch or semi-batch mode of operation. The operation of batch processes requires considerable attention, in particular regarding the coordination in time of various processing tasks such as charging, heating, reacting, separation, cooling, and the determination of optimal temperature and feeding profiles. Process control engineers have been eager to use their expertise in controlling and optimizing continuous processes to achieve comparable success with batch processes. However, this is rarely possible, the reason being significant differences between continuous and batch processes. The two main distinguishing features are discussed first ${ }^{2}$ :

- Distinguishing feature \#1: No steady-state operating point. In batch processes, chemical and physical transformations proceed from an initial state to a very different final state. In a batch reactor, for instance, even if the reactor temperature is kept constant, the concentrations, and thus also the reaction rates, change significantly over the duration of the batch. Consequently, there does not exist a steadystate operating point around which the control system can be designed. The decision variables are infinite-dimensional time profiles. Furthermore, important process characteristics — such as static gains, time constants and time delays - are time varying.

- Distinguishing feature \#2: Repetitive nature. Batch processing is characterized by the frequent repetition of batch runs. Hence, it is appealing to use the results from previous runs to optimize the operation of subsequent ones. This has generated the industrially relevant topics of run-to-run control and run-to-run optimization. 
In addition to these two features, a number of issues tend to complicate the operation of batch processes:

- Nonlinear behavior. Constitutive equations such as reaction rates and thermodynamic relationships are typically nonlinear. Since a batch process operates over a wide range of conditions, it is not possible to use, for the purpose of control design and optimization, models that have been linearized around a steady-state operating point, as this is typically done for continuous processes.

- Poor models. There is little time in batch processing for thorough investigations of the reaction, mixing, heat- and mass-transfer issues. Consequently, the models are often poor. For example, it may well happen that, in the production of specialty chemicals, the number of significant reactions is unknown, not to mention their stoichiometry or kinetics.

- Few specific measurements. The sensors that allow measuring concentrations online are rare. Chemical composition is usually determined by drawing a sample and analyzing it offline, that is, by using invasive and destructive methods. Furthermore, the available measurements - often physical quantities such as temperature, pressure, torque, turbidity, reflective index and electric conductivity — might exhibit low accuracy due to the wide range of operation that the measuring instrument has to cover.

- Constrained operation. A process is typically designed to operate in a limited region of the state space. In addition, the values that the inputs can take are upper and lower bounded. The presence of these limitations (labeled constraints) complicates the design of operational strategies for two main reasons: i) even if the process is linear or has been linearized along a reference trajectory, constraints make it nonlinear, and ii) controllability might be lost when a manipulated variable hits a constraint. Due to the wide operating range of batch processes, it is rarely possible to design the process so as to enforce feasible operation close to constraints, as this is typically done for continuous processes. In fact, it has been our experience that safety and operational constraints dominate the operation of batch processes.

- Presence of disturbances. Operator errors (e.g. wrong stirrer or solvent choice, incorrect material loading) and processing problems (e.g. fouling of sensors and reactor walls, insufficient mixing, incorrect feeding profiles, sensor failures) represent major disturbances that, unfortunately, cannot be totally 
ruled out. There are other unmeasured disturbances entering the process as the result of upstream process variability such as impurities in the raw materials.

- Irreversible behavior. In processes with history-dependent product properties, such as polymerization and crystallization, it is often impossible to introduce remedial corrections once off-specification material has been produced. This contrasts with continuous processes, where appropriate control action can bring the process back to the desired steady state following an upset in operating conditions.

- Limited corrective action. The ability to influence the process typically decreases with time. This, together with the finite duration of a batch run, limits the impact of corrective actions. Often, if a batch run shows a deviation in product quality, the charge has to be discarded.

\section{Models of Batch Processes}

Model-based control and optimization techniques rely on appropriate mathematical representations. The meaning of the qualifier "appropriate" depends on the system at hand and the objectives of the study. Some modeling aspects of batch processes are addressed next ${ }^{3}$.

\subsection{What to Model?}

Consider the example of a batch chemical reactor. The reactor system comprises the reactor vessel and one or several reactions. For reasons mentioned above, the reactions are often poorly known in the batch environment. Even when the desired reaction and the main side reactions are well documented, there might exist additional poorly known or totally unknown reactions. For example, it is frequently observed that the desired products and the known side products do not account for all the transformed reactants. Hence, since the stoichiometry of the reaction system is not completely known, the kinetic models are often of aggregate nature, that is, they encompass real and pseudo (lumped) reactions in order to describe the observed concentrations. This way, the number of modeled reactions, and thereby the number of kinetic 
parameters to be estimated, can be kept low. Furthermore, to determine the operational strategy for a reactor, it is necessary to consider the reaction kinetics, reactor dynamics and operational constraints. In industrial situations, the dynamics associated with the thermal exchange between the reactor and the jacket are often dominant and must necessarily be included in the model. The reasoning developed here for batch reactors ${ }^{4,5}$ extends similarly to other batch units such as batch distillation columns ${ }^{6,7}$ and batch crystallizers $^{8,9}$.

\section{$3.2 \quad$ Model Types}

The models used can be of several types, the characteristics of which are detailed next.

- Data-driven black-box models. Despite their simplicity, empirical input-output models are often able to satisfactorily represent the relationship between manipulated and observed variables. Linear and nonlinear ARMAX-type models are readily used to represent dynamic systems ${ }^{10,11}$. Neural network methods have also been proposed to model the dynamics of batch processes ${ }^{12}$. More recently, researchers have investigated the use of multivariate statistical partial least-squares (PLS) models as a means to predict product quality in batch reactors ${ }^{13}$. Experimental design techniques can help assess and increase the validity of data-driven models ${ }^{14}$. Although simple and relatively easy to obtain, data-driven input-output models have certain drawbacks:

1. They often exhibit good interpolative capabilities, yet they are inadequate for predicting the process behavior outside the experimental domain in which the data were collected for model building. Such a feature significantly limits the applicability of these models for optimization, that is, the determination of better profiles that have not been seen before.

2. Input-output models represent a dynamic relationship only between variables that are manipulated or measured. Unfortunately, some key variables, such as concentrations, often remain unmeasured in batch processes and thus cannot be modeled via data-driven models.

- Knowledge-driven white-box models. A mechanistic state-space representation based on energy and 
material balances is the preferred approach for modeling batch processes. The rate expressions describe the effect that the temperature and the concentrations have on the various rates. The model of a given unit relates the independent variables (inputs and disturbances) to the states (concentrations, temperature and volume) and the corresponding outputs.

The dynamic model of a batch process can be written as follows ${ }^{15}$ :

$$
\begin{aligned}
\dot{x}_{k}(t) & =F\left(x_{k}(t), u_{k}(t)\right), \quad x_{k}(0)=x_{0, k}, \\
y_{k}(t) & =H\left(x_{k}(t), u_{k}(t)\right), \\
z_{k} & =Z\left(x_{k}\left[0, t_{f}\right], u_{k}\left[0, t_{f}\right]\right),
\end{aligned}
$$

where $t$ denotes the run time, $k$ the batch or run index, $x$ the $n$-dimensional state vector, and $u$ the $m$ dimensional input vector. There are two types of measured outputs, namely, the $p$-dimensional vector of run-time outputs $y(t)$ that are available online and the $q$-dimensional vector of run-end outputs $z$ that are available at the final time $t_{f}$. Note that $z$ is not limited to quantities measured at the final time but can include quantities inferred from the profiles $x_{k}\left[0, t_{f}\right]$ and $u_{k}\left[0, t_{f}\right]$, such as the maximal temperature reached during the batch.

In addition to the run-time dynamics specific to a given run, one has the possibility of updating the initial conditions and the inputs on a run-to-run basis as follows:*

$$
\begin{aligned}
x_{0, k+1} & =I\left(x_{k}\left[0, t_{f}\right], u_{k}\left[0, t_{f}\right]\right), \quad x_{0,0}=x_{\text {init }}, \\
u_{k+1}\left[0, t_{f}\right] & =K\left(x_{k}\left[0, t_{f}\right], u_{k}\left[0, t_{f}\right]\right), \quad u_{0}\left[0, t_{f}\right]=u_{\text {init }}\left[0, t_{f}\right] .
\end{aligned}
$$

Note that, in this formulation, the final time $t_{f}$ is the same for all batches.

Mechanistic models are typically derived from physico-chemical laws. They are well suited for a wide range of process operations. However, they are difficult and time-consuming to build for industrially relevant processes. A sensitivity analysis can help evaluate the dominant terms in a model and retain those that are most relevant to the processing objectives ${ }^{16}$. No realistic model is purely mechanistic, as a few physical parameters typically need to be estimated from process data. As with data-driven

*The initial conditions and inputs can be updated on the basis of several prior batches; the relationships proposed here consider only the previous batch for simplicity of notation. 
models, experimental design techniques ${ }^{17}$ are useful tools for building sound models from a limited amount of data.

- Hybrid grey-box models. As a combination of the two extreme cases listed above, hybrid models can be very helpful in certain situations. They typically possess a simple structure that is based on some qualitative knowledge of the process ${ }^{18}$. The model parameters are particularly easy to identify if the model structure can be reduced to an ARMAX-type form ${ }^{19}$. For example, reaction lumping is often exercized. Because of the aggregate structure of the model, it is necessary to adjust certain model parameters online as, for example, the rate parameters might represent a different aggregation initially than toward the end of the batch. This can be done continuously or in some ad-hoc fashion, but clearly at a rate slower than that of the main dynamics. Furthermore, tendency models have been proposed ${ }^{20}$. These models retain the physical understanding of the system but are expressed in a form that is suitable to be fitted to local behavior. Although tendency modeling appears to be of high industrial relevance, it has received little attention in the academic research community.

\subsection{Static View of a Batch Process}

Due to the absence of a steady state, most signals in a batch process evolve with time. Consequently, the decision variables (here the input profiles $u\left[0, t_{f}\right]$ for a given batch run) are of infinite dimension. It is however possible to represent a batch process as a static map between a finite number of input parameters (used to define the input profiles before batch start) and the run-end outputs (representing the outcome at batch end). The key element is the parameterization of the input profiles as

$$
u\left[0, t_{f}\right]=\mathcal{U}(\pi, t)
$$

with the input parameters $\pi$. This parameterization is achieved by dividing the input profiles into time intervals and using a polynomial approximation (of which the simplest form is a constant value) within each interval. The input parameter vector $\pi$ can also include switching times between intervals ${ }^{21}$.

With such a parameterization, the batch process can be seen as a static map between the finite set 
of input parameters $\pi$ and the run-end outputs $z$ :

$$
z=M(\pi)
$$

This static model indicates that, once the input parameters $\pi$ have been specified, it is possible to compute the run-end outputs $z$. For this, one needs to generate the input profiles $u\left[0, t_{f}\right]$ using Eq. (6), integrate Eq. (1) and generate $z$ via Eq. (3). Alternatively, with an experimental set-up, one can generate the input profiles $u\left[0, t_{f}\right]$, apply them to the batch process and collect information regarding the run-end outputs $z$. Although the static model (7) looks simple, one has to keep in mind that the batch dynamics are hidden by the fact that only the relationship between the input parameters (before the run) and the run-end outputs (after the run) are considered. The dynamics are re presented implicitly in the static map between $\pi$ and $z$. These two different ways of looking at the operation of a given batch are illustrated in Figure 1 . Very naturally, the "Dynamic view" will be relevant for online operations, while the "Static view" will be more suited for run-to-run operations.

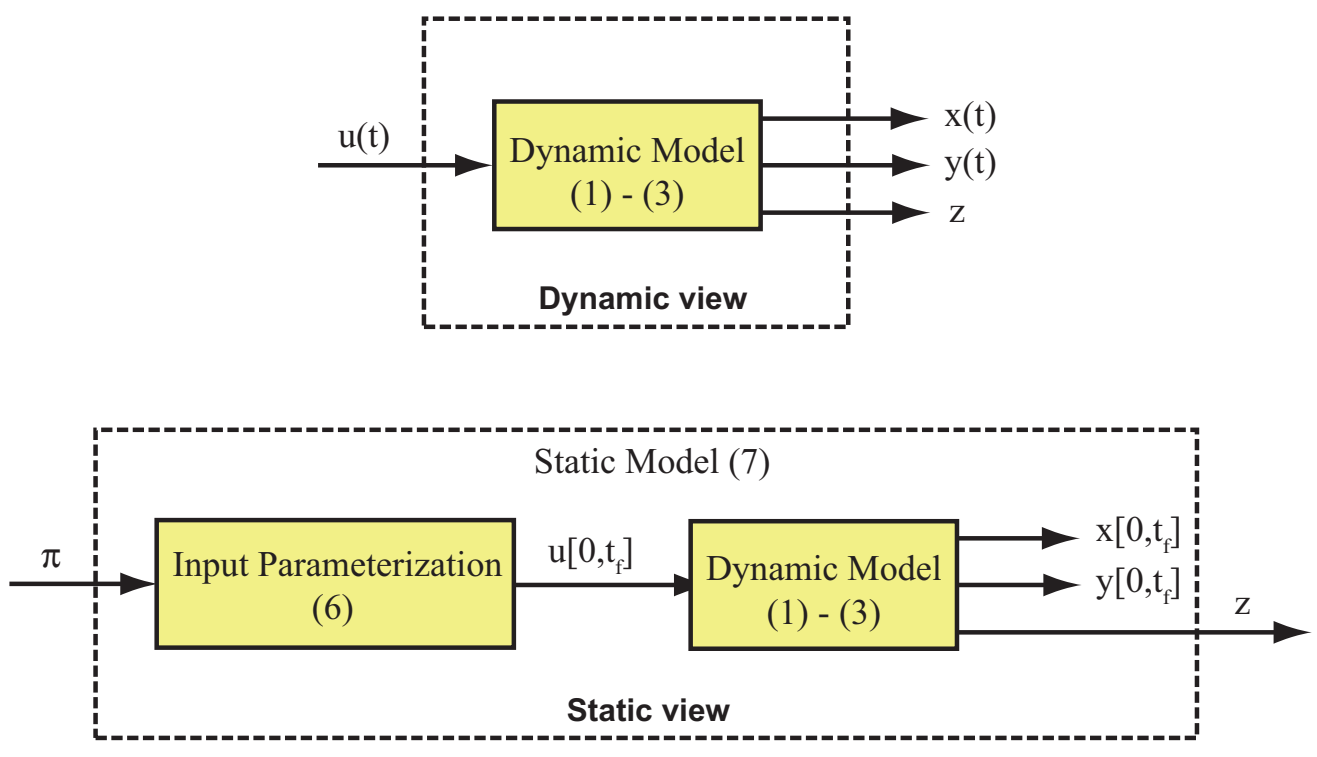

Figure 1: Two different views of the same batch run. Dynamic view: the dynamic model describes the behavior of the states $x(t)$, the run-time outputs $y(t)$ and the run-end outputs $z$ as functions of the inputs $u(t)$ Static view: the static model $z=M(\pi)$ explicits the relationship between the input parameters $\pi$ and the run-end outputs $z$. 


\section{PART A. CONTROL}

Batch process control has gained popularity over the past forty years. This is due to the development of novel algorithms with proven ability to improve the performance of industrial processes despite uncertainty and perturbations. But the development of new sensors and of automation tools also contributed to this success. At this stage, it is important to distinguish between control and automation. In practice, industry often tends to consider that control and automation are two identical topics and use the two words interchangeably. Although arguable, we will consider hereafter that control corresponds to the theory, that is, the research field, whereby new control methods, algorithms and controller structures are sought. On the other hand, automation represents the technology (the set of devices and their organization) that is needed to program and implement control solutions.

The control of batch processes differs from the control of continuous processes because of the two main distinguishing features presented in Section 2. First, since batch processes have no steady-state operating point, the setpoints to track are time-varying trajectories. Second, batch processes are repeated over time and are characterized by two independent variables, the run time $t$ and the run index $k$. The independent variable $k$ provides additional degrees of freedom for meeting the control objectives when these objectives do not necessarily have to be completed in a single batch but can be distributed over several successive batches. Batch process control aims to track two types of references:

a. The run-time references $y_{r e f}(t)$ or $y_{r e f}\left[0, t_{f}\right]$ are determined offline either from accumulated experience and knowledge of the process, or by solving a dynamic optimization problem (see Section 8.1). Examples of trajectories to be tracked include concentration, temperature, pressure, conversion and viscosity profiles. In general, trajectory tracking can be achieved in two ways: (i) via "online control" in a single batch, whereby input adjustments are made as the batch progresses based on online measurements, or (ii) via "run-to-run control" over several batches, where the input trajectories are computed between batches using information gathered from previous batches. It is also possible to combine approaches (i) and (ii) as will be detailed later. 
b. The run-end references $z_{r e f}$ are associated with the run-end outputs that are only available at the end of the batch. The most common run-end outputs are product quality, productivity and selectivity. As with run-time references, the run-end references can be tracked online or on a run-to-run basis.

With these two types of control objectives and the two different ways of reaching them (online and over several runs), there are four different control strategies as illustrated in Figure 2. It is interesting to see what view (dynamic or static) each control strategy uses. It turns out that Strategies 1-3 use a dynamic view of the batch process, with only Strategy 4 based on the static view. In the following, Section 4 addresses online control (the first row in Figure 1, Strategies 1 and 2), while Section 5 deals with run-to-run control (the second row in Figure 1, Strategies 3 and 4).

\section{Online Control}

The idea of "online control" is to take corrective action during the batch, that is, in run-time $t$. It is possible to do so with two objectives in mind, namely, the run-time reference trajectories $y_{r e f}(t)$ and the run-end references $z_{\text {ref }}$. This will be discussed in the next two subsections.

\subsection{Feedback Control of Run-time Outputs (Strategy 1)}

We will address successively the application of conventional feedback control to batch processes, the thermal control of batch reactors, and the stability issue for batch processes.

\subsubsection{Conventional feedback control}

The application of online feedback control to batch processes has the peculiarity that the setpoints are often time-varying trajectories. Even if some of the controlled variables, such as the temperature in isothermal operation, remain constant, the key process parameters (static gains, time constants and time delays) can 


\begin{tabular}{|c|c|c|}
\hline \multirow{2}{*}{$\begin{array}{l}\text { Implementation } \\
\text { aspect }\end{array}$} & \multicolumn{2}{|c|}{ Control objective } \\
\hline & $\begin{array}{l}\text { Run-time references } \\
y_{\text {ref }}(t) \text { or } y_{\text {ref }}\left[0, t_{f}\right]\end{array}$ & $\begin{array}{c}\text { Run-end references } \\
z_{r e f}\end{array}$ \\
\hline $\begin{array}{c}\text { Online } \\
-->u(t), u\left[t, t_{f}\right]\end{array}$ & $\begin{array}{l}\text { (1) Feedback control } \\
u(t) \longrightarrow \mathrm{P} \longrightarrow y(t)\end{array}$ & 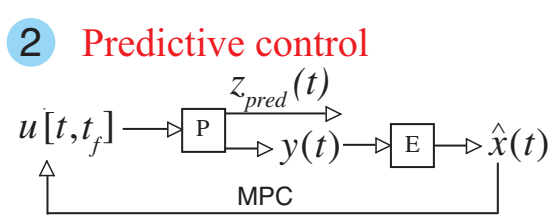 \\
\hline $\begin{array}{l}\text { Run-to-run } \\
-->u\left[0, t_{f}\right]\end{array}$ & $\begin{array}{l}3 \text { Iterative learning control } \\
u\left[0, t_{f}\right] \longrightarrow \mathrm{P} \longrightarrow y\left[0, t_{f}\right] \\
\text { ILC with run update }\end{array}$ & $\begin{array}{l}\text { (4) Run-to-run control } \\
\pi \stackrel{\mathcal{U}(\pi)}{\longrightarrow} u\left[0, t_{f}\right] \longrightarrow \mathrm{P} \longrightarrow z \\
\text { RiR with run update }\end{array}$ \\
\hline
\end{tabular}

Figure 2: Control strategies for batch processes. The strategies are classified according to the control objective (horizontal division) and the implementation aspect (vertical division). Each objective can be met either online or on a run-to-run basis depending on the type of measurements available. The signal $u(t)$ represents the input vector at time $t, u\left[0, t_{f}\right]$ the corresponding input trajectories, $y(t)$ the run-time outputs measured online at time $t$, and $z$ the run-end outputs available at the end of the batch. P stands for "plant", E for "state estimator", FbC for "feedback control", MPC for "model predictive control", ILC for "iterative learning control", and RtR for "run-to-run control". When online and run-to-run control are combined, the run-to-run part $u\left[0, t_{f}\right]$ can be considered as the feedforward contribution, while the online part $u(t)$ is the feedback part. 
vary considerably during the duration of the batch.

Feedback control is implemented using PID techniques or more sophisticated alternatives such as cascade control, predictive control, disturbance compensation (feedforward control) and time-delay compensation $^{22,23}$. The online feedback controller can be written formally as

$$
u(t)=\mathcal{K}\left(y(t), y_{\text {ref }}(t)\right)
$$

where $\mathcal{K}$ is the online control law for run-time outputs as illustrated in Figure 3.

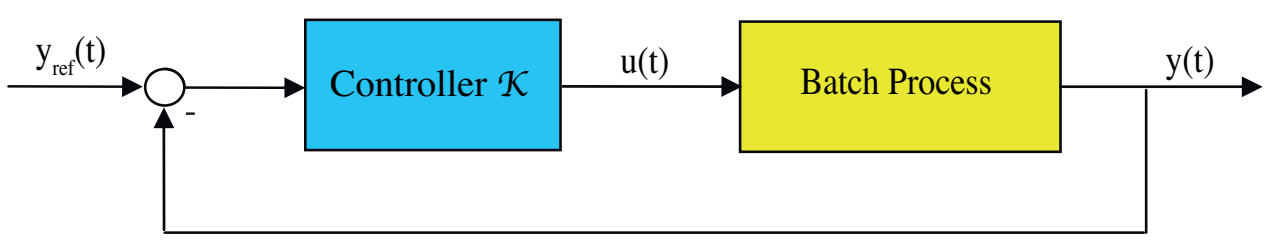

Figure 3: Online feedback control of the run-time outputs $y$ in a batch process.

One way to adapt for variations in process characteristics is via adaptive control ${ }^{24}$. However, adaptive control requires continuous process identification, which is often impractical in batch processes due to the finite batch duration. Instead, practitioners use conventional feedback control and schedule the values of some of the controller parameters (mostly the gains, thus leading to gain scheduling ${ }^{25}$ ). Appropriate values of the control parameters can be computed offline ahead of time for the various phases of the batch run and stored for later use.

A few industrial applications of advanced feedback control are available in the literature. For example, a successful implementation of temperature control in an industrial $35-m^{3}$ semi-batch polymerization reactor using a flatness-based two-degree-of-freedom controller has been reported ${ }^{26}$. Also, the performance of four different controllers (standard PI control, self-tuning PID control, and two nonlinear controllers) for regulating the reactor temperature in a 5-liter jacketed batch suspension methyl methacrylate polymerization reactor has been compared ${ }^{27}$. As expected, the performance of the standard PI controller was the poorest since the controller parameters were fixed and not adapted to changing process characteristics. The selftuning PID control performed better because available measurements were used to adapt the controller to 
the varying process characteristics. The two nonlinear controllers, which were based on differential-geometric techniques requiring full-state measurement ${ }^{28}$, showed excellent performance despite significant uncertainty in the heat-transfer coefficient.

\subsubsection{Control of heat generation}

The control of exothermic reactions in batch reactors is a challenging problem that is tackled using one of various operation modes ${ }^{29}$ :

1) Isothermal batch operation. Most reaction systems are investigated isothermally in the laboratory. For safety and selectivity reasons, pilot-plant and industrial reactors are also run isothermally at the same temperature specifications. However, the transfer function between the jacket inlet temperature and the reactor temperature depends on the amount of heat evolved, and it can become unstable for certain combinations of the reaction parameters ${ }^{29}$. Adaptation of the controller parameters is therefore necessary. Most often, this is done by using pre-computed values, that is, via gain scheduling. An energy balance around the reactor is sometimes used to estimate the heat generated by the chemical reactions, which represents the major disturbance for the control system. This estimate can then be used very effectively in a feedforward scheme. It is important to mention that isothermal batch operation often exhibits low productivity because it is limited by the maximal heat-generation rate (corresponding to the maximal heat-removal capacity of the reactor vessel), which typically occurs initially for only a short period of time.

2) Isothermal semi-batch operation. The productivity of isothermal batch reactors can be increased through semi-batch operation. This way, the reactant concentrations can be increased once the initial phase characterized by the heat-removal limitation is over. For strongly exothermic reactions, semi-batch operation has the additional advantage of improving the effective heat-removal capacity through feeding of cold reactants. This, in turn, increases the productivity of the reactor by allowing higher temperatures. Hence, isothermal semi-batch operation is often the preferred way of running discontinuous reactors in industry. The temperature is controlled by manipulating the feedrate of the limiting reactant. However, the 
system exhibits non-minimum phase behavior, that is, a flowrate increase of the cold feed first reduces the temperature before the temperature raises due to higher reaction rates, which significantly complicates the control $^{30}$. Furthermore, in most semi-batch operations, it is useful to finish off with a batch phase so as to fully consume the limiting reactant. Without a kinetic model, it is not obvious when to switch from the semi-batch to the batch mode. Finally, although isothermal, the operation of a semi-batch reactor should be such that the heat-removal constraint is never violated. This has forced industrial practice to be rather conservative, with a temperature setpoint often chosen from adiabatic considerations.

3) Constant-rate batch operation. Since a key objective in the operation of batch reactors is to master the amount of heat produced by the chemical transformations, it is meaningful to maintain a constant reaction rate. The temperature is kept low initially when the concentrations are high, to be increased with time as the reactants deplete. This way, the reactor productivity is considerably higher than through isothermal operation. However, large temperature excursions can have a detrimental effect on product selectivity. If reaction kinetics are known, it is a simple matter to compute the temperature profile that results in the desired heat generation. If the heat evolution can be measured or estimated online, feedback control can maintain it at the (constant) setpoint. Laboratory-scale reaction calorimeters have been devised to measure or estimate the heat-evolution profiles of (possibly unknown) reaction systems ${ }^{31}$. The temperature profiles obtained in the laboratory can then be used in production environments, at least in qualitative terms, provided that sufficient heat-removal capacity is available.

\subsubsection{Run-time stability of batch processes}

Stability is of uppermost importance to prevent runaway-type behavior. However, run-time asymptotic stability is of little value since $t_{f}$ is finite. Hence, one can say that "instability" can be tolerated for a batch process because of its finite duration.

Yet, an important issue in run time is reproducibility, which addresses the question of whether the trajectories of various runs with sufficiently close initial conditions and identical input profiles will remain 
close during the run. The problem of stability is in fact related to the sensitivity with respect to perturbations. The basic framework for assessing this sensitivity is as follows:

- the system is perturbed by either variations of the initial conditions or external disturbances that affect the system states,

- sensitivity is assessed as a norm indicating the relative effect of the perturbations.

For a system to be stable, this norm must remain bounded for a bounded perturbation, and it must go to zero with time for a vanishing perturbation. However, when dealing with finite-time systems, it is difficult to infer stability from this norm since, except for some special cases such as finite escape time, boundedness is guaranteed. Also, the vanishing behavior with time cannot be analyzed since $t_{f}$ is finite. The element of interest is in fact the numerical value of the norm, and the issue becomes quantitative rather than binary (yes-no). Details can be found elsewhere ${ }^{15}$.

\subsection{Predictive Control of Run-end Outputs (Strategy 2)}

With a sufficiently accurate process model, and in the absence of disturbances, tracking the profiles determined offline is often sufficient to meet the batch-end product quality requirements ${ }^{32}$. However, in the presence of disturbances, following pre-specified profiles is unlikely to lead to the desired product quality. Hence, the following question arises: Is it possible to design an online control scheme for effective control of run-end outputs using run-time measurements? Since such an approach amounts to controlling a quantity that has not yet been measured, it is necessary to predict run-end outputs to compute the required corrective control action. Model predictive control (MPC) is well suited for the task of controlling future quantities that need to be predicted ${ }^{33}$. This approach to batch control can, therefore, be formulated as an MPC problem with a shrinking prediction horizon (equal to the remaining duration of the batch) and an objective function that penalizes deviations from the desired product quality at batch end ${ }^{34}$. 


\subsubsection{Model predictive control}

With the MPC strategy, two steps need to be implemented online at each sampling time, namely, the estimation of the current states and the computation of piecewise-constant profiles of future control moves.

First step: Online estimation of $x(t)$. This is done via state estimation using Model (1)-(2) and measurements of the run-time outputs $y(t)^{35,36}$. The resulting state estimate is denoted $\hat{x}(t)$. However, this task is difficult to implement with sufficient accuracy because of some of the limiting characteristics of batch processes, in particular their nonlinear behavior, the presence of large model inaccuracies and the lack of specific measurements.

$\underline{\text { Second step: Online prediction of } z \text { and computation of } u\left[t, t_{f}\right]}$. In the context of batch operation, predictive control consists in determining the inputs $u\left[t, t_{f}\right]$ to meet $z_{\text {ref }}$ by solving at time $t$ a finite-horizon open-loop optimal control problem. Concretely, we consider the dynamic model given by Eqns (1) and (3) and solve the following optimization problem at the discrete time instant $t_{i}$ :

$$
\begin{aligned}
\min _{u\left[t_{i}, t_{f}\right]} & J:=\frac{1}{2} \Delta z^{T}\left(t_{i}\right) P \Delta z\left(t_{i}\right)+\frac{1}{2} \int_{t_{i}}^{t_{f}} u^{T}(t) R u(t) d t \\
\text { s.t. } & \dot{x}(t)=F(x(t), u(t)), \quad x\left(t_{i}\right)=\hat{x}\left(t_{i}\right), \\
& z_{\text {pred }}\left(t_{i}\right)=Z\left(x\left[0, t_{f}\right], u\left[0, t_{f}\right]\right), \\
& x\left(t_{f}\right) \in \mathcal{X},
\end{aligned}
$$

where $z_{\text {pred }}\left(t_{i}\right)$ are the run-end outputs predicted at time $t_{i}, \Delta z\left(t_{i}\right):=z_{\text {pred }}\left(t_{i}\right)-z_{\text {ref }}$ the predicted run-end errors, $P$ and $R$ are positive-definite weighting matrices of appropriate dimensions, $\mathcal{X}$ is the bounded region of state space where the final state should lie, $t_{i}$ is the current discrete time instant at which the optimization is performed, and $t_{f}$ the final time. Note that the profiles $u\left[0, t_{f}\right]$ and $x\left[0, t_{f}\right]$ include (i) the parts $u\left[0, t_{i}\right]$ and $x\left[0, t_{i}\right]$ that were determined prior to the time $t_{i}$, and (ii) the the parts $u\left[t_{i}, t_{f}\right]$ and $x\left[t_{i}, t_{f}\right]$ that are computed at $t_{i}$. The integral term in the objective function (9) is optional and serves to penalize large control efforts. Numerical optimization yields the control sequence, $u^{*}\left[t_{i}, t_{f}\right]$, of which only the first part, $u^{*}\left[t_{i}, t_{i}+h\right]$, is applied to the plant in an open-loop fashion, where $h$ is the sampling period. Numerical optimization is then repeated at every sampling instant. Note that it is important to include a bounded 
region for the final states for the sake of stability ${ }^{33}$.

The control law can be written formally as

$$
u\left[t, t_{f}\right]=\mathcal{P}\left(z_{\text {pred }}(t), z_{\text {ref }}\right)
$$

where $\mathcal{P}$ is the predictive control law for run-end outputs and $z_{\text {pred }}(t)$ is the prediction of $z$ available at time $t$ as illustrated in Figure 4.

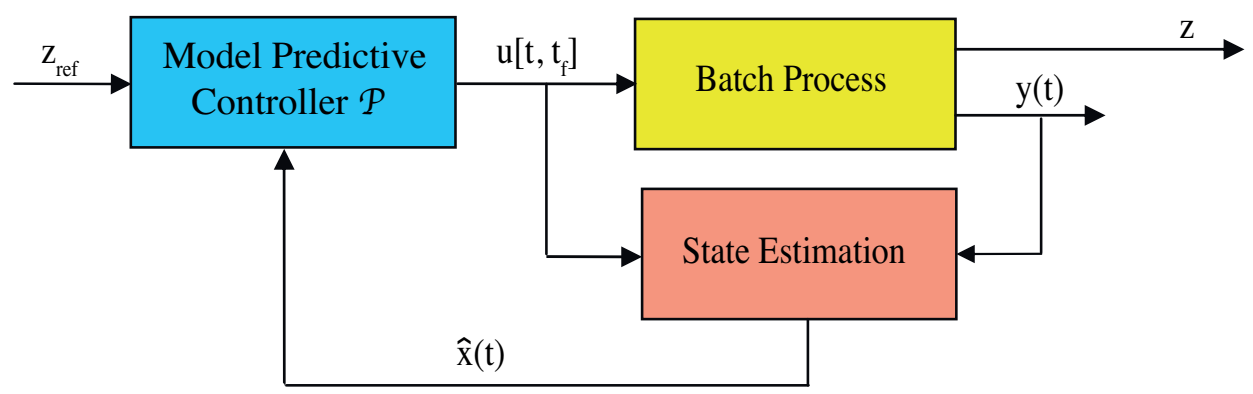

Figure 4: Online predictive control of the run-end outputs $z$ in a batch process. The model in the "Model Predictive Controller" block is used to compute $z_{\text {pred }}(t)$ from $\hat{x}(t)$.

Linear MPC is widely used in industry, more so than nonlinear MPC. However, one may ask whether linear MPC is sufficient to deal with highly nonlinear batch processes? Note that nonlinear MPC is an active area of research. For example, a nonlinear MPC scheme has been proposed to regulate run-end molecular properties within bounds in the context of batch polymerization reactors ${ }^{37}$.

\subsubsection{Alternative ways of meeting $z_{r e f}$ online}

In MPC, $z$ is predicted online as $z_{\text {pred }}(t)$ from the state estimates $\hat{x}(t)$, and then the control profile $u^{*}\left[t, t_{f}\right]$ is computed via numerical optimization. This is rather demanding, in particular regarding the availability of an accurate process model for both state estimation and input computation. This requirement can be reduced significantly with the approaches proposed next.

Use of data-driven PLS models. When the detailed dynamic model (1) is not available, it is possible to use partial least-squares (PLS) regression models identified from historical data and a few additional experi- 
ments $^{38}$. Then, in real time during the progress of the batch, it is possible to use the PLS model to estimate the run-end outputs and track $z_{r e f}$ by adjusting $u\left[t, t_{f}\right]$ via run-to-run control (see Section 5.2).

Another less ambitious but very efficient control approach has also been proposed, whereby, if the prediction of $z$ falls outside a predefined no-control region, a mid-course correction is implemented ${ }^{39}$. Note that the approach can easily be modified to accommodate more than one mid-course correction.

Still another variant of the use of PLS models involves controlling the process in the reduced space (scores) of a latent variable model rather than in the space of the discretized inputs ${ }^{40}$.

Use of online tracking. An alternative for meeting run-end references using online measurements consists in tracking some feasible trajectory, $z_{r e f}(t)$, whose main purpose is to enforce $z_{r e f}$ at final time, that is, $z_{r e f}\left(t_{f}\right)=z_{r e f}{ }^{41}$. This necessitates to be able to measure or estimate $z(t)$ during the batch.

\section{Run-to-run Control}

Run-to-run control takes advantage of the repetition of batches that is characteristic of batch processing. One uses information from previous batches to improve the performance of the next batch. This can be achieved with respect to both run-time and run-end objectives, as discussed in the next two subsections.

\subsection{Iterative Learning Control of Run-time Profiles (Strategy 3)}

The time profiles of the manipulated variables can be generated using Iterative Learning Control (ILC), which exploits information from previous runs to improve the performance of the current run ${ }^{42}$. This strategy exhibits the limitations of open-loop control with respect to the current run, in particular the fact that there is no feedback correction for run-time disturbances. Nevertheless, this scheme is useful for generating time-varying feedforward input terms. The ILC controller has the formal structure

$$
u_{k+1}\left[0, t_{f}\right]=\mathcal{I}\left(y_{k}\left[0, t_{f}\right], y_{r e f}\left[0, t_{f}\right]\right)
$$


where $\mathcal{I}$ is the ILC law for run-time outputs. ILC uses the entire profiles of the previous run to generate the input profiles for the next run as illustrated in Figure 5. ILC has been successfully applied in robotics ${ }^{43,44}$ and in batch chemical processing ${ }^{45,46}$.

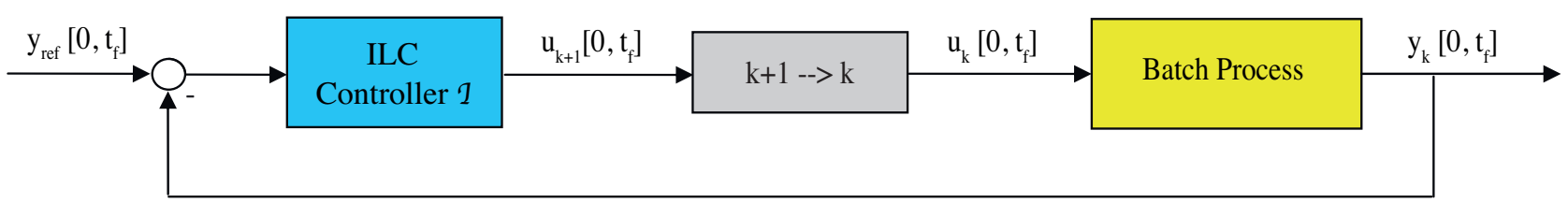

Figure 5: ILC of the run-time profiles $y_{k}\left[0, t_{f}\right]$ in a batch process. The run update is indicated by $k+1 \rightarrow k$.

\subsubsection{ILC problem formulation}

A repetitive batch process is considered in operator notation:

$$
y_{k}\left[0, t_{f}\right]=\mathcal{G}\left(u_{k}\left[0, t_{f}\right], x_{k}(0)\right)
$$

where $u_{k}\left[0, t_{f}\right]$ and $x_{k}(0)$ represent the input profiles and the initial conditions in run $k, \mathcal{G}$ is the operator representing the system. This relationship can be obtained from Model (1)-(2) as follows:

- With the inputs $u_{k}\left[0, t_{f}\right]$ and the initial conditions $x_{k}(0)$ specified, integrate Eq. (1) to obtain $x_{k}\left[0, t_{f}\right]$.

- Eq. (2) allows computing $y_{k}\left[0, t_{f}\right]$ from $x_{k}\left[0, t_{f}\right]$ and $u_{k}\left[0, t_{f}\right]$.

For the case where the system operator is linear and the signals are in discrete time, Eq. (15) can be expressed in matrix form as follows:

$$
y_{k}=G u_{k}+y_{0, k},
$$

with $y_{k} \in \Re^{N p}, u_{k} \in \Re^{N m}$ and $G \in \Re^{N p \times N m}$, where $N$ is the finite number of time samples, $p$ the number of outputs, and $m$ the number of inputs. $y_{0, k} \in \Re^{N p}$ represents the response to the initial conditions $x_{k}(0)$.

ILC tries to improve trajectory following by utilizing the previous-cycle tracking errors. The ILC update law for the inputs $u_{k+1}$ is given as

$$
u_{k+1}=A u_{k}+B e_{k}, \quad e_{k}=y_{r e f}-y_{k},
$$


where $y_{\text {ref }} \in \Re^{N p}$ are the references to be tracked, and $A \in \Re^{N m \times N m}$ and $B \in \Re^{N m \times N p}$ are operators applied to the previous-cycle inputs and tracking errors, respectively. In the remainder of this section dealing with ILC, the signals without an explicit time dependency are expressed as vectors containing the $N$ finite time samples, that is, $u_{k} \in \Re^{N m}, y_{k} \in \Re^{N p}$ and $e_{k} \in \Re^{N p}$.

\subsubsection{ILC convergence and residual errors}

An ILC law is convergent if the following limits exist:

$$
\lim _{k \rightarrow \infty} y_{k}=y_{\infty} \quad \text { and } \quad \lim _{k \rightarrow \infty} u_{k}=u_{\infty}
$$

One way to ensure convergence is that the relation between $u_{k}$ and $u_{k+1}$ be a contraction mapping in some appropriate norm:

$$
\left\|u_{k+1}\right\| \leq \rho\left\|u_{k}\right\|, \quad 0 \leq \rho<1 .
$$

Using (16) in Eq. (17) gives:

$$
u_{k+1}=(A-B G) u_{k}+B\left(y_{r e f}-y_{0, k}\right) .
$$

Note that $\left(y_{r e f}-y_{0, k}\right)$ does not represent errors as in Eq. (17), but the differences between the reference trajectories and the responses to the initial conditions. Also, in contrast to Eq. (17), where $e_{k}$ depends on $u_{k}$ through $y_{k},\left(y_{r e f}-y_{0, k}\right)$ is independent of $u_{k}$. Hence, the convergence of the algorithm depends on the homogenous part of Eq. (20) and the condition for convergence is:

$$
\|A-B G\|<1
$$

If the iterative scheme converges, with $u_{k}=u_{k+1}=u_{\infty}$ and $y_{0, k}=y_{0, k+1}=y_{0}$, Eq. (20) gives:

$$
u_{\infty}=(I-A+B G)^{-1} B\left(y_{r e f}-y_{0}\right)
$$

Since the converged outputs $y_{\infty}$ are not necessarily equal to the desired outputs $y_{r e f}$, the final tracking errors can be different from zero. The final tracking errors are given by

$$
e_{\infty}=y_{r e f}-\left(G u_{\infty}+y_{0}\right)=\left(I-G(I-A+B G)^{-1} B\right)\left(y_{r e f}-y_{0}\right)
$$


If $B G$ is invertible, this equation can be rewritten as

$$
e_{\infty}=G(B G)^{-1}(I-A)(I-A+B G)^{-1} B\left(y_{r e f}-y_{0}\right)
$$

Note that the residual errors are zero when $A=I$ and non zero otherwise. Hence, the choice $A=I$ provides integral action along the run index $k$. Zero errors imply that perfect system inversion has been achieved.

\subsubsection{ILC with current-cycle feedback}

In conventional ILC schemes, only the tracking errors of the previous cycle are used to adjust the inputs in the current cycle. To be able to reject within-run perturbations, the errors occurring during the current cycle can be used as well, which leads to modified update laws. However, from the points of view of convergence and error analysis, these modified schemes simply correspond to different choices of the operators $A$ and $B$ in Eq. (17) as shown next. Let

$$
\begin{aligned}
u_{k+1} & =u_{k+1}^{f f}+u_{k+1}^{f b}, \\
\text { with } \quad u_{k+1}^{f f} & =\bar{A} u_{k}^{f f}+\bar{B} e_{k}, \quad u_{k+1}^{f b}=C e_{k+1},
\end{aligned}
$$

where the superscripts $(\cdot)^{f f}$ and $(\cdot)^{f b}$ are used to represent the feedforward and feedback parts of the inputs, and $\bar{A}, \bar{B}$ and $C$ are operators.

Using Eq. (26) in Eq. (25) and combining it with Eqns (16)-(17) gives:

$$
\begin{aligned}
u_{k+1} & =(I+C G)^{-1}(\bar{A}(I+C G)-\bar{B} G) u_{k}+(I+C G)^{-1}(\bar{B}+C-\bar{A} C)\left(y_{\text {ref }}-y_{0}\right) \\
& =(A-B G) u_{k}+B\left(y_{r e f}-y_{0}\right)
\end{aligned}
$$

where $A=(I+C G)^{-1}(\bar{A}+C G)$ and $B=(I+C G)^{-1}(\bar{B}+(I-\bar{A}) C)$. The convergence condition and residual errors can be analyzed using the operators $A$ and $B$, similarly to what was done in Section 5.1.2.

\subsubsection{ILC with improved performance}

The standard technique for approximate inversion is to introduce a forgetting factor in the input update ${ }^{47}$. This causes the residual tracking errors to be non zero over the entire interval. Note, however, that the 
main difficulty with the feasibility of inversion arises during the first part of the trajectory due to unmatched initial conditions ${ }^{48}$. After a certain catch-up time, trajectory following is relatively easy. Hence, the idea is to allow non-zero tracking errors early in the run and have the errors decrease progressively with run time $t$, which can be achieved with an input shift. This is documented next as part of an ILC implementation that includes (i) input shift, (ii) shift of the previous-cycle errors, and (iii) current-cycle feedback ${ }^{49}$.

The iterative update law is written as follows:

$$
u_{k+1}(t)=u_{k+1}^{f f}(t)+K^{f b} e_{k+1}(t)
$$

where $K^{f b} \in \Re^{N m \times N p}$ are the proportional gains of the online feedback controller. The feedforward part of the current inputs, $u_{k+1}^{f f}(t)$, consists of shifted versions of the feedforward part of the previous inputs and the previous-cycle tracking errors:

$$
u_{k+1}^{f f}(t)=u_{k}^{f f}\left(t+\delta_{u}\right)+K^{f f} e_{k}\left(t+\delta_{e}\right)
$$

where $K^{f f} \in \Re^{N m \times N p}$ are the proportional gains of the feedforward controller, $\delta_{u}$ the time shift of the feedforward input trajectories and $\delta_{e}$ the time shift of the previous-run error trajectory. The remaining parts of the inputs and errors are kept constant, that is, $u_{k}^{f f}\left[t_{f}-\delta_{u}, t_{f}\right]=u_{k}^{f f}\left(t_{f}-\delta_{u}\right)$ and $e_{k}\left[t_{f}-\delta_{e}, t_{f}\right]=e_{k}\left(t_{f}-\delta_{e}\right)$, respectively.

\subsection{Run-to-run Control of Run-end Outputs (Strategy 4)}

In this case, the interest is to steer the run-end outputs $z$ towards $z_{\text {ref }}$ using the input parameters $\pi$. For this, one uses the static model (7) that results from the input parameterization (6). The run-to-run feedback control law can be written as

$$
\begin{aligned}
\pi_{k+1} & =\mathcal{R}\left(z_{k}, z_{\text {ref }}\right), \\
u_{k}\left[0, t_{f}\right] & =\mathcal{U}\left(\pi_{k}, t\right),
\end{aligned}
$$


where $\mathcal{U}$ represents the input parameterization and $\mathcal{R}$ is the run-to-run feedback control law for run-end outputs as illustrated in Figure 6. For example, one can use the discrete integral control law

$$
\pi_{k+1}=\pi_{k}+K\left(z_{r e f}-z_{k}\right)
$$

where $K$ is an appropriate gain matrix. To be able to meet exactly all the references, it is necessary to have at least as many inputs parameters as there are run-end outputs.

Static Map

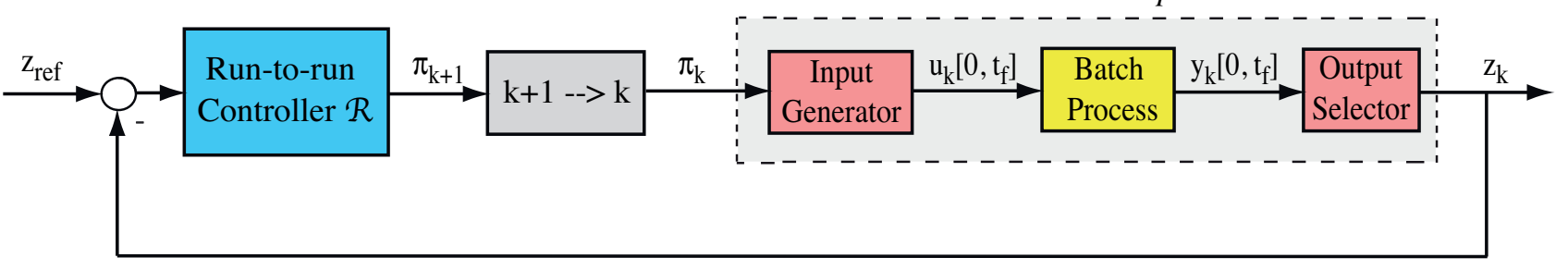

Figure 6: Run-to-run control of the run-end outputs $z$ in a batch process. The input generator constructs $u_{k}\left[0, t_{f}\right]$ from $\pi_{k}$ according to Eq. (31), while the output selector selects the run-end outputs.

\subsubsection{Control algorithm}

Run-to-run control proceeds as follows:

1. Initialization. Parameterize the input profiles $u(t)$ as $\mathcal{U}(\pi, t)$ in such a way that the input parameter vector $\pi$ has the same dimension as the vector of run-end outputs $z$ in order to generate a square control problem. Set $k=1$ and select an initial guess for $\pi_{k}$.

2. Iteration. Construct the inputs $u_{k}\left[0, t_{f}\right]$ using Eq. (31) and apply them to the process. Complete the run and determine $z_{k}$ from the measurements.

3. Update the input parameters using the control law (30). Set $k:=k+1$ and repeat Steps 2-3 until convergence. 


\subsubsection{Convergence analysis}

The convergence of the run-to-run algorithm can be determined by analyzing the closed-loop error dynamics, where the errors are $e_{k}:=z_{r e f}-z_{k}$.

We discuss next a convergence analysis that considers a linearized version of System (7) and the linear integral control law (32). With the linear model,

$$
z_{k}=S \pi_{k}
$$

where $S=\left.\frac{\partial M}{\partial \pi}\right|_{\bar{\pi}}$ is the sensitivity matrix computed at the nominal operating point corresponding to $\bar{\pi}$, the linearized error dynamics become:

$$
e_{k+1}=e_{k}-S K e_{k}=(I-S K) e_{k}
$$

The eigenvalues of the matrix $(I-S K)$ should be within the unit circle for the algorithm to converge. Convergence is determined by both the sensitivity matrix $S$ and the controller gains $K$. Convergence analysis of run-to-run control algorithms is also possible when the relationship between $\pi_{k}$ and $z_{k}$ is nonlinear, but it typically requires additional assumptions regarding the nature of the nonlinearities ${ }^{50}$.

\subsubsection{Run-to-run stability}

The interest in studying stability in the run index $k$ arises from the necessity to guarantee convergence of run-to-run control schemes. Here, the standard notion of stability applies as the independent variable $k$ goes to infinity. The main conceptual difference with the stability of continuous processes is that "equilibrium" refers to entire trajectories. Hence, the norms have to be defined in the space of functions $\mathcal{L}$ such as the integral squared norm $l_{2}$ of the signal $x(t)$ :

$$
\left\|x\left[0, t_{f}\right]\right\|_{l_{2}}=\int_{0}^{t_{f}}\left(x(t)^{2} d t\right)^{1 / 2} .
$$

For studying stability with respect to the run index $k$, System (1) is considered under closed-loop operation, that is, with all possible online and run-to run feedback loops. Hence, we no longer restrict our 
attention to the input-output run-to-run $\pi-z$ behavior, but we consider the entire state vector. For simplicity, let us assume that $t_{f}$ is constant for all runs. When dealing with the $k^{t h}$ run, the trajectories of the $(k-1)^{s t}$ run are known, which fixes $u_{k}\left[0, t_{f}\right]$ according to the ILC control law (14). These input profiles, along with the online feedback law (8) or (13), are applied to System (1) to obtain $x_{k}(t)$ for all $t$ and thus $x_{k}\left[0, t_{f}\right]$. All these operations can be represented formally as:

$$
x_{k}\left[0, t_{f}\right]=\mathcal{F}\left(x_{k-1}\left[0, t_{f}\right]\right), \quad x_{0}\left[0, t_{f}\right]=x_{\text {init }}\left[0, t_{f}\right]
$$

where $x_{\text {init }}\left[0, t_{f}\right]$ are the initial state trajectories obtained by integration of Eq. (1) for $k=0, x_{0,0}=x_{\text {init }}$ and $u_{0}\left[0, t_{f}\right]=u_{i n i t}\left[0, t_{f}\right]$. Eq. (36) describes the run-to-run dynamics associated with all control activities. Run-to-run stability is considered around the equilibrium trajectory computed from $(36)$, that is $\bar{x}\left[0, t_{f}\right]=$ $\mathcal{F}\left(\bar{x}\left[0, t_{f}\right]\right)$, and is investigated using a Lyapunov-function method ${ }^{51}$.

\section{Batch Automation}

Although batch processing was dominant until the 30 's, batch process control received significant interest only 50 years later ${ }^{52}$. This is mainly due to the fact that, when control theory started to emerge in the 40 's, there was a shift from the dominance of batch processing to continuous operations. Hence, the dynamics and control of batch processes became a subject of investigation in the 80's. Engineers then tried to carry over to batch processes the experience gained over the years with the control of continuous processes. However, the specificities of batch processes make their control quite challenging.

This is also true at the implementation level. If continuous processes typically exhibit four main regimes, namely, start-up, continuous operation, possibly grade transition and shut down, batch processes are much more versatile since, in the absence of a steady state, the system evolves freely from a set of initial conditions to a final state. During this transient operation, the recipe can be rather varied, ranging from basic isothermal operation to a succession of complex operations that can involve discrete decisions. Also, batch processes are often repeated over time, with or without changes between batches, which makes the task of designing a control strategy much more involved than for continuous operations. 
The control of batch operations relies on various types of controllers that include stand-alone controllers, programmable logic controllers (PLCs), distributed control systems (DCS), and personal computers (PCs). These four platforms are discussed next.

\subsection{Stand-alone Controllers}

The basic stand-alone controllers are single-loop controllers (SLCs) ${ }^{53}$. These devices generally embed a microprocessor with fixed functionalities such as PID control. The popularity of SLCs arose from their simplicity, low cost and small size. Originally, stand-alone controllers were capable of integrating dualloop control, that is, they can handle two control loops with basic on/off and PID control. More recently, these devices have incorporated self-tuning algorithms and, more importantly for batch processes, time scheduling and sequencing functionalities. Still, the main advantage of SLCs is their low cost per control loop. Furthermore, they are often used in parallel or in combination with more advanced control structures such as DCS, mainly because they can achieve very acceptable control performance for minimal investment regarding cost, maintenance and required knowledge.

\subsection{Programmable Logic Controllers}

PLCs were introduced in the process industries in the early 70's, following the development in the automotive industry, as computing systems "that had the flexibility of a computer, yet could be programmed and maintained by plant engineers and technicians" ${ }^{53}$. Before PLCs, relays, counters and timers were mainly used, but they offered much less flexibility. PLCs can perform complex computations as they have a computational power that is comparable to a small $\mathrm{PC}^{54}$.

Considerable progress was made with the introduction of micro-PLCs, which can be installed close to the process at a much lower cost. The development of Supervisory Control and Data Acquisition (SCADA) constitutes another breakthrough that has increased the scope of applications. It is possible to handle numerous operations distributed over a large distance, thereby opening up the applicability of PLCs to cases 
where batch process operations are fully integrated in the plant-wide operation of a production site.

\subsection{Distributed Control Systems}

DCS are control systems with control elements spread around the plant. They generally propose a hierarchical approach to control, with several interconnected layers operating at different time scales. At the top of the hierarchy is the production/scheduling layer, where the major decisions are taken on the basis of market considerations and measurements collected from the plant. These decisions are sent to an intermediate layer, where there are used, together with plant measurements and estimated fluctuations on price and raw-material quality, to optimize the plant performance. These decisions can take the form of setpoint trajectories for low-level controllers. This intermediate layer, which is often referred to as the real-time optimization layer, is implemented via DCS ${ }^{55}$. Because of their distributed nature, DCS are helpful to integrate batch operations into the continuous operation of a plant, which has considerable impact on the planning, scheduling and real-time optimization tasks ${ }^{56}$. The control and optimization hardware has improved continuously over the past decades, thereby keeping pace with the development of advanced control and optimization methods.

Although the differences between DCS and SCADA can appear to be subtle, one could state the following ${ }^{56}$ : DCS (i) are mainly process driven, (ii) are focused on small geographic areas, (iii) are suited to large integrated chemical plants, (iv) rely on good data quality, and (v) incorporate powerful closed-loop control hardware. On the other hand, SCADA is generally more "event driven", which makes it more suited to the supervision of multiple independent systems.

\subsection{Personal Computers}

PCs can also be used for the control and optimization of batch processes. It has been reported that $25 \%$ of batch process control involve personal computers. Of course, this figure does not mean that $25 \%$ of batch process control involves only PCs, as PCs are often used in combination with other controllers such as SLCs or PLCs. In industry, PCs are not only used to program and run control algorithms, but they include 
many other elements such as interfaces, communication protocols and networking ${ }^{53}$. The development of user-friendly software interfaces, of simulation tools, and of fast and reliable optimization software has been key to the development of batch processing. With the relatively low cost of PCs, it has become economically viable to use them routinely for control. While a PC gives a lot of flexibility, especially for taking educated decisions in the context of a multi-layered control structure, it can also be used as a stand-alone advanced controller or even as an online optimization tool. In practice, however, PC-based control is often limited to supervisory control, where the PC sends recommendations to low-level controllers. This can take the form of input profiles to implement or of output trajectories to track during batch operation. These recommendations can then be implemented directly by means of low-level controllers, or manually by an operator who can take the final decision of implementing or not the recommendations ${ }^{53}$.

\section{Control Applications}

\subsection{Control of Temperature and Final Concentrations in a Semi-batch Reactor}

\subsubsection{Reaction system}

Consider the reaction of pyrrole $A$ with diketene $B$ to produce 2 -acetoacetyl pyrrole $C$. Diketene is a very aggressive compound that reacts with itself and other species to produce the undesired products $D, E$, and $F^{57}$. The desired and side reactions are

$$
\begin{aligned}
A+B & \rightarrow C, \\
2 B & \rightarrow D, \\
C+B & \rightarrow E, \\
B & \rightarrow F .
\end{aligned}
$$

The reactions are highly exothermic, that is, they produce heat. The reactor is operated in semi-batch mode with $A$ present initially in the reactor and $B$ added with the feedrate profile $u(t)$. Moreover, the reaction 
system is kept isothermal by removing the heat produced by the chemical reactions through a cooling jacket surrounding the reactor. The flowrate of cooling fluid is adjusted to keep the reactor temperature at its desired setpoint.

\subsubsection{Model of the reactor}

The nonlinear dynamic model of the reaction system is obtained from a material balance for each species and a total mass balance ${ }^{58}$ :

$$
\begin{aligned}
\frac{d c_{A}}{d t} & =-\frac{u}{V} c_{A}-k_{1} c_{A} c_{B} \\
\frac{d c_{B}}{d t} & =\frac{u}{V}\left(c_{B}^{i n}-c_{B}\right)-k_{1} c_{A} c_{B}-2 k_{2} c_{B}^{2}-k_{3} c_{C} c_{B}-k_{4} c_{B} \\
\frac{d c_{C}}{d t} & =-\frac{u}{V} c_{C}+k_{1} c_{A} c_{B}-k_{3} c_{C} c_{B} \\
\frac{d c_{D}}{d t} & =-\frac{u}{V} c_{D}+k_{2} c_{B}^{2} \\
\frac{d c_{E}}{d t} & =-\frac{u}{V} c_{E}+k_{3} c_{C} c_{B} \\
\frac{d c_{F}}{d t} & =-\frac{u}{V} c_{F}+k_{4} c_{B} \\
\frac{d V}{d t} & =u
\end{aligned}
$$

where $c_{i}$ is the concentration of the $i^{t h}$ species, $V$ is the reactor volume, $k_{j}$ is the rate constant of the $j^{t h}$ reaction, $c_{B}^{i n}$ is the inlet concentration of $B$, and $u$ is the volumetric feedrate of $B$.

\subsubsection{Control objective}

One would like to operate the reactor safely and efficiently. If the heat produced cannot be removed by the cooling jacket, the reactions accelerate and produce even more heat. This positive feedback effect, known as thermal runaway, can result in high temperature and pressure and thus lead to an explosion; this is precisely what caused the Bhopal disaster ${ }^{59}$. The performance of the reactor is evaluated by its productivity, namely, the amount of desired product available at final time, as well as its selectivity, namely, the portion of converted reactant $B$ that forms the desired product $C$. Note that the performance criteria 
(productivity and selectivity) are evaluated at the final time, whereas the manipulated variable (feedrate of $B$ ) is a run-time profile. The control objective is to operate isothermally at $50^{\circ} \mathrm{C}$ and match the final concentrations $c_{B, r e f}$ and $c_{D, \text { ref }}$ obtained in the laboratory

\subsubsection{Online control, ILC and run-to run control}

The control strategies, illustrated in Figure 7, include (i) a cascade scheme for online feedback control of $T_{r}(t)$ and ILC update of $T_{j, r e f}^{f f}\left[0, t_{f}\right]$, and (ii) run-to-run control of the final concentrations through update of the input parameters $u_{1}$ and $u_{2}$.

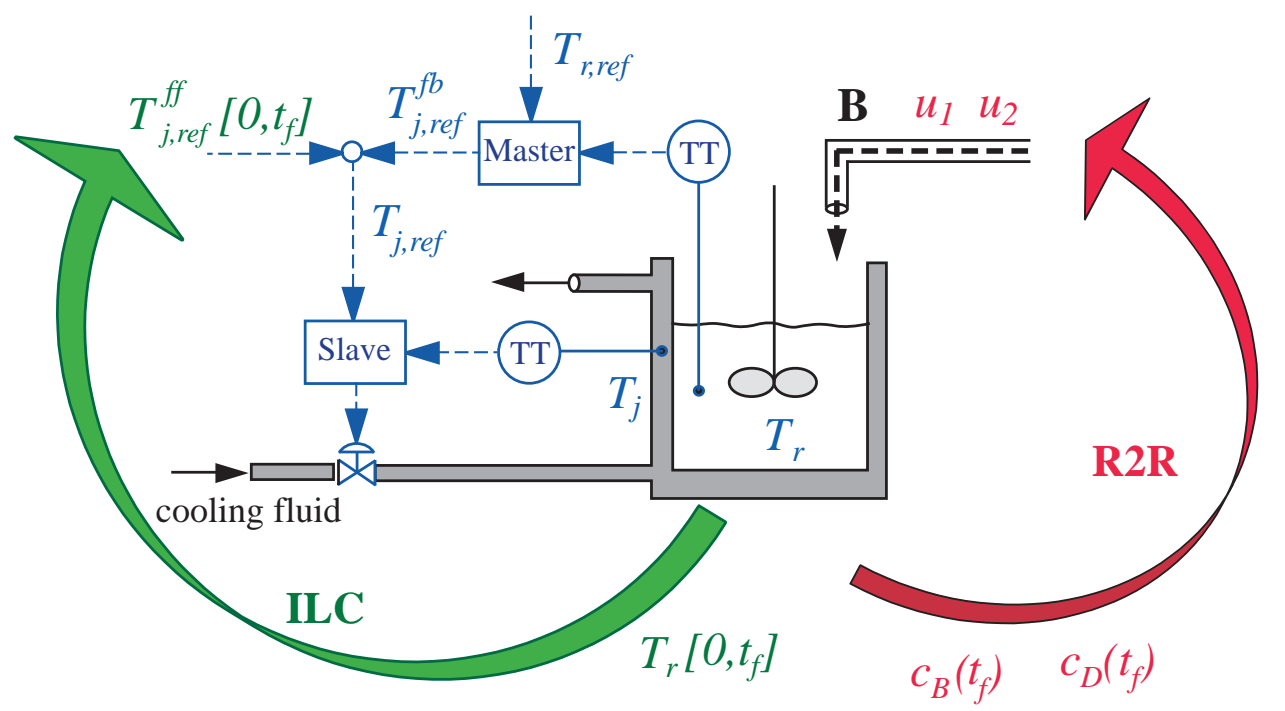

Figure 7: Online and run-to-run strategies for controlling the reactor temperature $T_{r}(t)$ and the final concentrations $c_{B}\left(t_{f}\right)$ and $c_{D}\left(t_{f}\right)$.

Control of the reactor temperature $T_{r}$ is implemented by means of a $\mathrm{PI} / \mathrm{P}$-cascade scheme that adjusts the flowrate of cooling fluid. The master loop contributes a feedback term to the reference value for the jacket temperature $T_{j}$. This reference value, which also includes a feedforward term, reads

$$
\begin{aligned}
& T_{j, r e f, k}(t)=T_{j, r e f, k}^{f f}(t)+T_{j, r e f, k}^{f b}(t), \\
& T_{j, r e f, k}^{f b}(t)=K_{R}\left(e_{k}(t)+\frac{1}{\tau_{I}} \int_{0}^{t} e_{k}(\tau) d \tau\right), \\
& T_{j, r e f, k+1}^{f f}\left[0, t_{f}\right]=T_{j, r e f, k}^{f f}\left[0, t_{f}\right]+K_{I L C} e_{k}\left[0, t_{f}\right],
\end{aligned}
$$


where $e_{k}(t):=T_{r, r e f}(t)-T_{r, k}(t), K_{R}$ is the proportional gain, and $\tau_{I}$ is the integral time constant of the PI master controller. $K_{I L C}$ is the gain of the ILC controller. Eq. (45) computes the feedback term $T_{j, r e f, k}^{f b}(t)$ using a PI feedback law, while Eq. (46) implements run-to-run adaptation of the feedforward term $T_{j, r e f, k}^{f f}(t)$ based on ILC.

The second manipulated variable is $u$, the feedrate of reactant $B$, through which the two reactions can be steered and brought to the desired final concentrations. Since these final concentrations are measured only at the end of the batch, the feedrate of $B$ is adjusted on a run-to-run basis. Two input parameters are needed to control these two concentrations. Hence, the feedrate profile $u\left[0, t_{f}\right]$ is parameterized using the two feedrate levels $u_{1}$ and $u_{2}$, each valid over half the batch time. The sensitivities of the final concentrations $z=\left(c_{B}\left(t_{f}\right), c_{D}\left(t_{f}\right)\right)^{T}$ with respect to $\pi=\left(u_{1}, u_{2}\right)^{T}$ is evaluated experimentally, which gives $S=\left.\frac{\partial z}{\partial \pi}\right|_{k}$. With this notation, the discrete integral control law has the form

$$
\pi_{k+1}=\pi_{k}+K_{R t R} S^{-1}\left[z_{r e f}-z_{k}\right]
$$

where $K_{R t R}$ is the $2 \times 2$ diagonal gain matrix of the run-to-run controller.

The contributions of ILC and run-to-run control to the control of reactor temperature and final concentrations are illustrated in Figures 8 and 9, respectively. One sees that adjustment of $T_{j, r e f}^{f f}$ using ILC reduces the maximal temperature excursion from $57.1^{\circ} \mathrm{C}$ to $52.7^{\circ} \mathrm{C}$.

\subsection{Scale-up via Feedback Control}

Short times to market are required in the specialty chemicals industry. One way to reduce this time to market is by skipping the pilot-plant investigations. However, due to scale-related differences in operating conditions, direct extrapolation of conditions obtained in the laboratory is often impossible, especially when terminal objectives must be met and path constraints respected. In fact, ensuring feasibility at the industrial scale is of paramount importance. This section presents an example for which the combination of online and run-to-run control allows meeting production requirements over a few batches. 

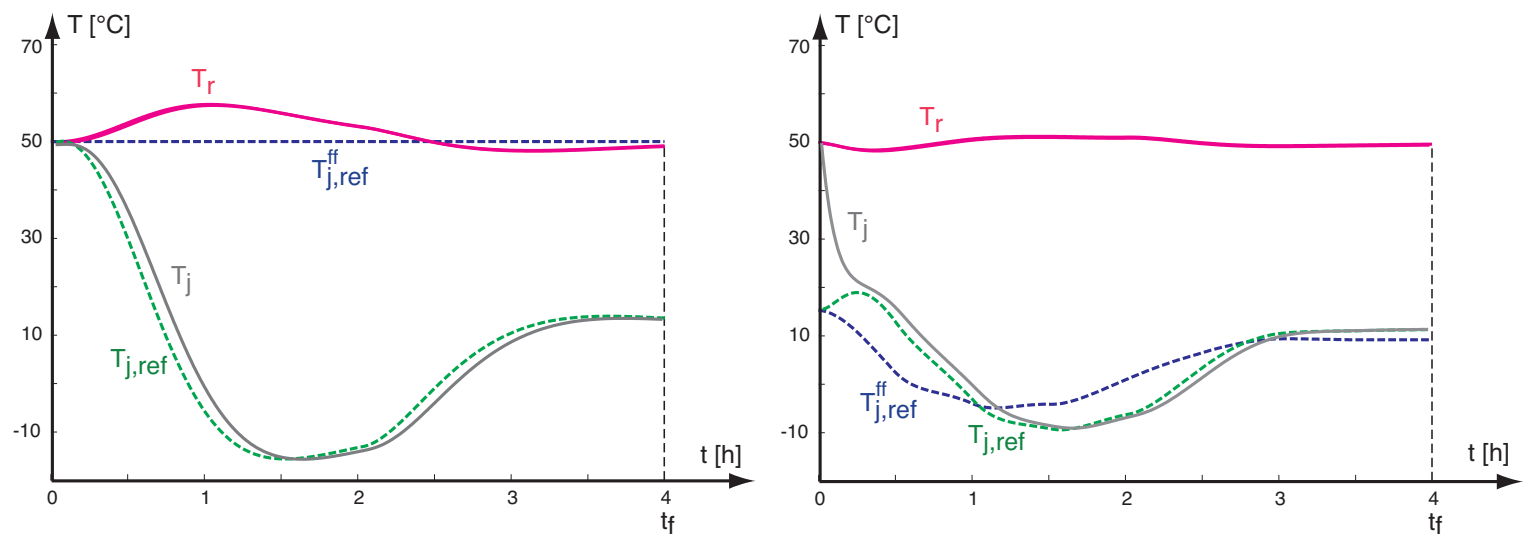

Figure 8: Temperature control around the constant setpoint $T_{r, r e f}=50^{\circ} \mathrm{C}$, without ILC (left), and after three ILC iterations (right). The red and grey solid lines represents the reactor temperature $T_{r}$ and the jacket temperature $T_{j}$, respectively. The blue and green dashed lines represent the feedforward term of the jacket temperature setpoint $T_{j, r e f}^{f f}$ and the resulting jacket temperature setpoint $T_{j, r e f}$, respectively.

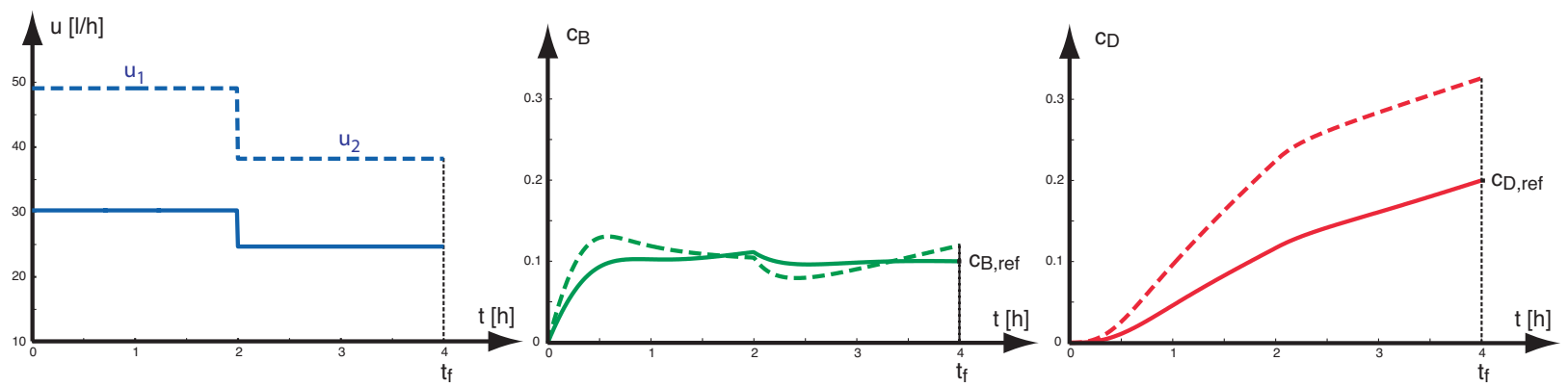

Figure 9: Run-to-run control for meeting the run-end concentrations $c_{B, r e f}$ and $c_{D, \text { ref }}$. The inlet feedrate is parameterized using the two levels $u_{1}$ and $u_{2}$ (left). The concentration profiles for $c_{B}(t)$ and $c_{D}(t)$ are shown in the center and at right, respectively. The dashed lines show the initial profiles, while the solid lines show the corresponding profiles after two iterations. The references $c_{B, r e f}$ and $c_{D, \text { ref }}$ are reached via run-to-run adjustment of $u_{1}$ and $u_{2}$. 


\subsubsection{Problem formulation}

Consider the following parallel reaction scheme ${ }^{60}$ :

$$
A+B \longrightarrow C, \quad 2 B \longrightarrow D
$$

The desired product is $C$, while $D$ is undesired. The reactions are exothermic. A 1-L reactor is used in the laboratory, while a jacketed reactor of $5 \mathrm{~m}^{3}$ is used in production. The manipulated inputs are the feedrate $F(t)$ and the coolant flowrate through the jacket $F_{j}(t)$. The operational requirements can be formulated as:

$$
\begin{gathered}
T_{j}(t) \geq 10^{\circ} \mathrm{C}, \\
y_{D}\left(t_{f}\right)=\frac{2 n_{D}\left(t_{f}\right)}{n_{C}\left(t_{f}\right)+2 n_{D}\left(t_{f}\right)} \leq 0.18,
\end{gathered}
$$

where $T_{j}(t)$ is the jacket temperature, and $n_{C}\left(t_{f}\right)$ and $n_{D}\left(t_{f}\right)$ denote the numbers of moles of $C$ and $D$ at final time.

\subsubsection{Laboratory recipe}

The recipe obtained in the laboratory proposes to initially fill the reactor with A, and then feed B at some constant feedrate $\bar{F}$, while maintaining the reactor isothermal at $T_{r}=40^{\circ} \mathrm{C}$. As cooling is not an issue for the laboratory reactor equipped with an efficient jacket, experiments were carried out using a scale-down approach, that is, the cooling rate was artificially limited so as to anticipate the limited cooling capacity of the industrial reactor. Scaling down is performed by the introduction of an operating constraint that limits the cooling capacity; in this case, the maximal cooling capacity of the industrial reactor is simply divided by the scale-up factor:

$$
\left[q_{c, \max }\right]_{\mathrm{lab}}=\frac{\left[\left(T_{r}-T_{j, \min }\right) U A\right]_{\mathrm{prod}}}{r}
$$

where $r=5000$ is the scale-up factor and $U A=3.7 \times 10^{4} \mathrm{~J} / \mathrm{mol}{ }^{\circ} \mathrm{C}$ is the estimated heat-transfer capacity of the production reactor. With $T_{r}-T_{j, \min }=30^{\circ} \mathrm{C}$, the maximal cooling rate is $222 \mathrm{~J} / \mathrm{min}$. Table 1 summarizes the key parameters of the laboratory recipe and the (simulated) laboratory results. 
Table 1: Laboratory recipe and results for the scale-up problem.

\begin{tabular}{|c|c|c|}
\hline \multicolumn{2}{|c|}{ Recipe parameters } & Laboratory results \\
\hline$T_{r}=40^{\circ} \mathrm{C}$ & $c_{B, i n}=5 \mathrm{~mol} / \mathrm{L}$ & $n_{C}\left(t_{f}\right)=0.346 \mathrm{~mol}$ \\
\hline$c_{A, o}=0.5 \mathrm{~mol} / \mathrm{L}$ & $c_{B, o}=0 \mathrm{~mol} / \mathrm{L}$ & $y_{D}\left(t_{f}\right)=0.170$ \\
\hline$V_{0}=1 \mathrm{~L}$ & $t_{f}=240 \mathrm{~min}$ & $\max _{t} q_{c}(t)=182.6 \mathrm{~J} / \mathrm{min}$ \\
\hline $\bar{F}=4 \times 10^{-4} \mathrm{~L} / \mathrm{mi}$ & & \\
\hline
\end{tabular}

\subsubsection{Scale-up via online control, ILC and run-to run control}

The goal of scale-up is to reproduce in production the productivity and selectivity that are obtained in the laboratory, while enforcing the desired reactor temperature. With selectivity and productivity as run-end outputs, the feedrate profile $F\left[0, t_{f}\right]$ is parameterized using two adjustable parameters, namely, the constant feedrate levels $F_{1}$ and $F_{2}$, each one valid over half the batch time. Temperature control is done via a combined feedforward/feedback scheme as illustrated in Section 7.1.

A control problem can be formulated with the following manipulated variables (MVs), controlled variables $(\mathrm{CVs})$ and setpoints (SPs):

$$
\begin{aligned}
& \text { - MVs: } u(t)=T_{j, r e f}(t), \quad \pi=\left[\begin{array}{ll}
F_{1}, & F_{2}
\end{array}\right]^{T} \\
& \text { - CVs: } y(t)=T_{r}(t), \quad z=\left[\begin{array}{ll}
n_{C}\left(t_{f}\right), & y_{D}\left(t_{f}\right)
\end{array}\right]^{T} \\
& \text { - SPs: } \quad y_{\text {ref }}=40^{\circ} \mathrm{C}, \quad z_{\text {ref }}=\left[\begin{array}{ll}
1630 \mathrm{~mol}, & 0.17
\end{array}\right]^{T} \text {. }
\end{aligned}
$$

The setpoints are chosen as follows:

- $T_{r, r e f}=40^{\circ} \mathrm{C}$ is the value used in the laboratory.

- $n_{C, \text { ref }}$ is the value obtained in the laboratory multiplied by the scale-up factor $r$ minus a 100-mol backoff to account for scale-related uncertainties and run-time disturbances. The introduction of backoff makes the control problem more flexible.

- $y_{D, \text { ref }}$ is the value obtained in the lab, which respects the upper bound of 0.18 . 
The control scheme is shown in Figure 10. The input profiles are updated using (i) the cascade feedback controller $\mathcal{K}$ to control the reactor temperature $T_{r}(t)$ online, (ii) the ILC controller $\mathcal{I}$ to improve the reactor temperature by adjusting $T_{j, r e f}^{f f}(t)$, and (iii) the run-to-run controller $\mathcal{R}$ to control $z=\left[n_{C}\left(t_{f}\right), y_{D}\left(t_{f}\right)\right]^{T}$ by adjusting $\pi=\left[\begin{array}{ll}F_{1}, & F_{2}\end{array}\right]^{T}$. Details regarding the implementation of the different control elements can be found elsewhere ${ }^{60}$.

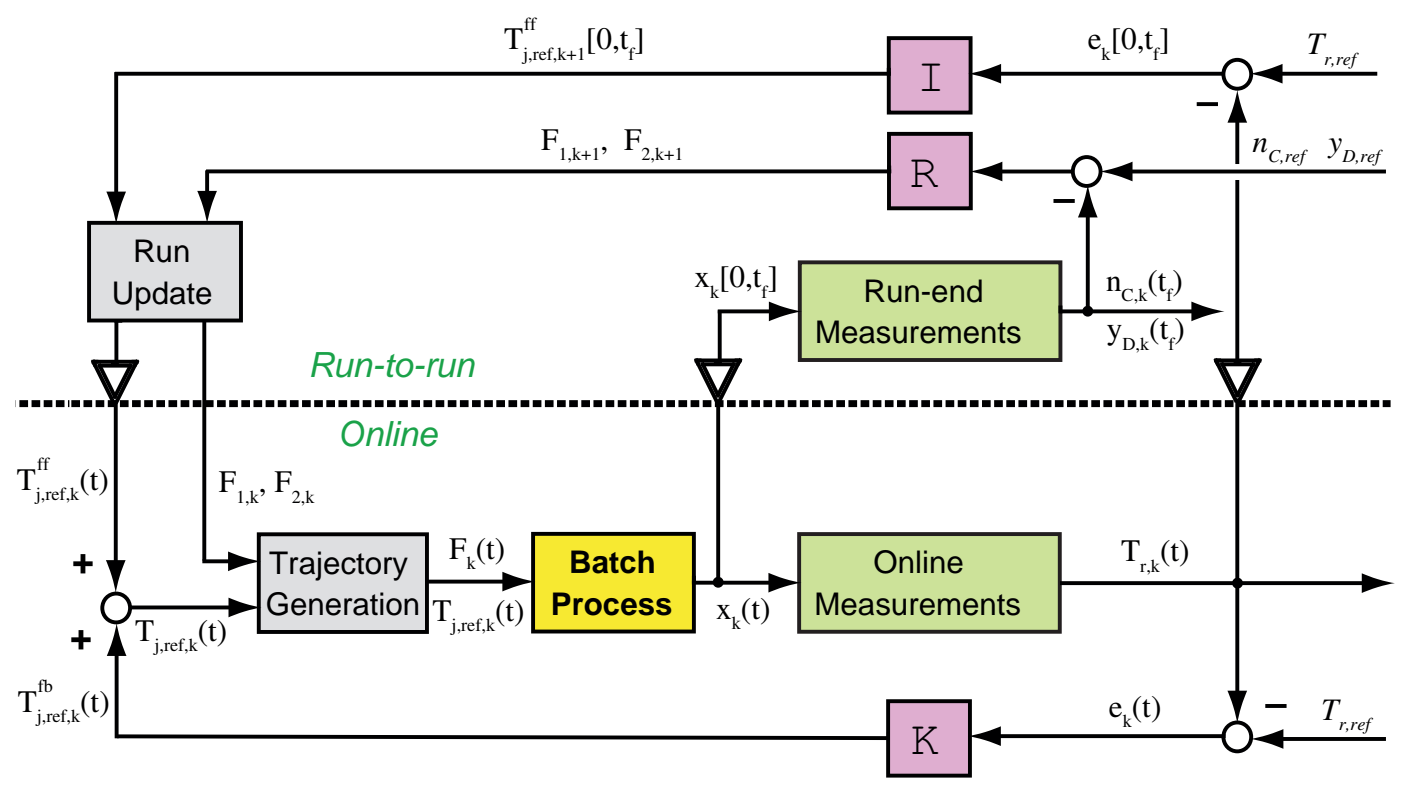

Figure 10: Control scheme for scale-up implementation. Notice the distinction between online and run-torun activities. The symbol $\nabla$ represents the concentration/expansion of information between a profile (e.g. $\left.x_{k}\left[0, t_{f}\right]\right)$ and an instantaneous value (e.g. $\left.x_{k}(t)\right)$.

\subsubsection{Simulation results}

The recipe presented in Table 1 is applied to the $5-\mathrm{m}^{3}$ industrial reactor, equipped with a $2.5-\mathrm{m}^{3}$ jacket. In this simulated case study, uncertainty is introduced artificially by modifying the two kinetic parameters, which are reduced by $25 \%$ and $20 \%$, respectively. Also, Gaussian noise with standard deviations of $0.001 \mathrm{~mol} / \mathrm{L}$ and $0.1{ }^{\circ} \mathrm{C}$ is considered for the measurement of the final concentrations and for the reactor temperature, respectively. It turns out that, for the first run, application of the laboratory recipe with 
$\pi=\left[\begin{array}{rl}r \bar{F}, & r \bar{F}\end{array}\right]^{T}$ violates of the final selectivity of $\mathrm{D}$ in the first batch. Upon adapting the MVs with the proposed scale-up algorithm, the free parts of the recipe are modified iteratively to achieve the production targets for the industrial reactor, as illustrated in Figure 11.

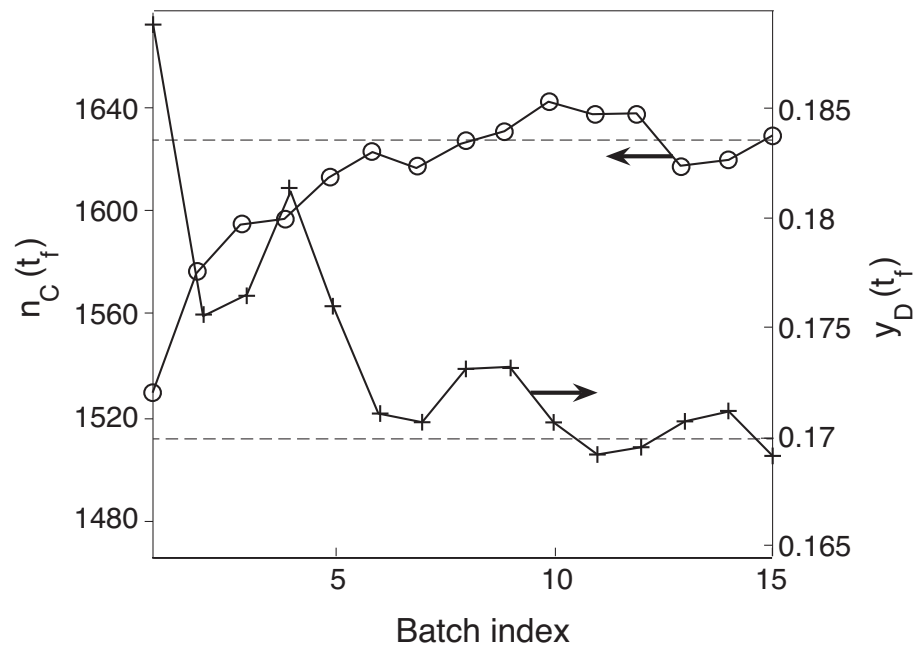

Figure 11: Evolution of the production of $C, n_{C}\left(t_{f}\right)$, and the yield of $D, y_{D}\left(t_{f}\right)$, for the large-scale industrial reactor. Most of the improvement is achieved in the first 7 runs (the dashed lines represent the target values).

\subsection{Control of a Batch Distillation Column}

A binary batch distillation column is used to illustrate in simulation the application of iterative learning control. This example is described in detail elsewhere ${ }^{61}$.

\subsubsection{Model of the column}

A binary batch distillation column with $p$ equilibrium stages is considered (see Figure 12). Using standard assumptions ${ }^{62}$ and writing molar balance equations for the holdup in the reboiler and for the liquid on the 


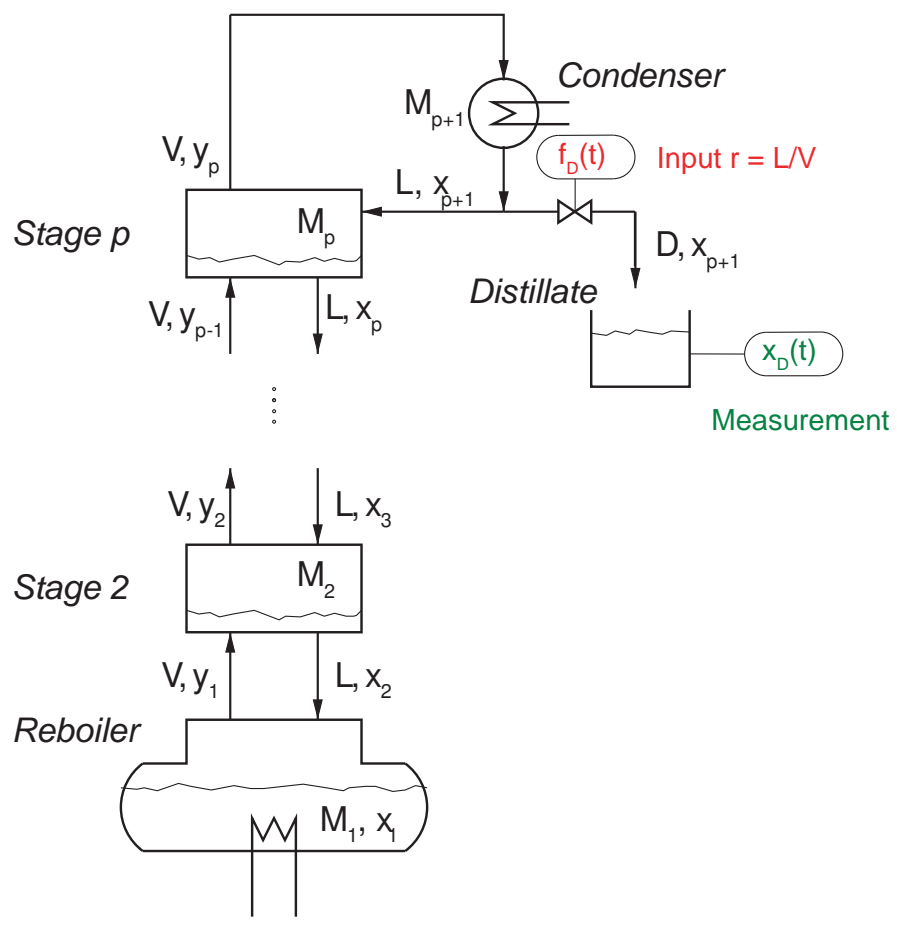

Figure 12: Binary batch distillation column.

various stages and in the condenser, the following model of order $(p+2)$ is obtained:

$$
\begin{aligned}
\dot{M}_{1} & =(r-1) V \\
\dot{x}_{1} & =\frac{V}{M_{1}}\left(x_{1}-y_{1}+r x_{2}\right), \\
\dot{x}_{i} & =\frac{V}{M_{i}}\left(y_{i-1}-y_{i}+r\left(x_{i+1}-x_{i}\right)\right), \quad i=2, \ldots, p, \\
\dot{x}_{p+1} & =\frac{V}{M_{p+1}}\left(y_{p}-x_{p+1}\right),
\end{aligned}
$$

where $x_{i}$ is the molar liquid fraction, $y_{i}$ the molar vapor fraction, $M_{i}$ the molar holdup on Stage $i, V$ the vapor flowrate, and $D$ the distillate flowrate. Stage 1 refers to the reboiler, Stage $p$ to the top of the column, and Stage $p+1$ to the condenser. The internal reflux ratio $r=\frac{L}{V}=\frac{V-D}{V}$ is considered as the manipulated variable. The vapor-liquid equilibrium relationship is:

$$
y_{i}=\frac{\alpha x_{i}}{1+(\alpha-1) x_{i}}, \quad i=1, \cdots, p
$$


where $\alpha$ is the relative volatility. The composition of the accumulated distillate, $x_{D}$, which is measured with the sampling time $h=0.1 h$, is given by:

$$
x_{D}(t)=\frac{\sum_{i=1}^{p} x_{i}(t) M_{i}(t)-x_{i}(0) M_{i}(0)}{M_{1}(t)-M_{1}(0)} .
$$

The model parameters, the initial conditions and the control objective $x_{D, \text { ref }}$ are given in Table 2 .

Table 2: Model parameters and initial conditions, $i=2, \cdots, p$.

\begin{tabular}{ccc|ccc}
\hline$p$ & 10 & & $M_{1}(0)$ & 100 & $\mathrm{kmol}$ \\
$\alpha$ & 1.6 & & $x_{1}(0)$ & 0.5 & \\
$M_{i}$ & 0.2 & $\mathrm{kmol}$ & $x_{i}(0)$ & 0.5 & \\
$M_{p+1}$ & 2 & $\mathrm{kmol}$ & $x_{p+1}(0)$ & 0.5 & \\
$V$ & 15 & $\mathrm{kmol} / \mathrm{h}$ & $x_{D, r e f}$ & 0.9 & \\
$t_{f}$ & 10 & $\mathrm{~h}$ & $h$ & 0.1 & $\mathrm{~h}$ \\
\hline
\end{tabular}

\subsubsection{Operational objective}

A batch is divided into 2 operational phases:

1. Start-up phase with full reflux up to time $t_{s}: r=1, t=\left[0, t_{s}\right], t_{s}=1.415 \mathrm{~h}$.

2. Distillation phase: $r \in[0,1], t=\left(t_{s}, t_{f}\right], t_{f}=10 \mathrm{~h}$.

The objective is to adjust the reflux ratio to get the distillate purity $x_{D}\left(t_{f}\right)=0.9$ at the end of the batch. This will be obtained by tracking an appropriate reference trajectory for the distillate purity $x_{D}(t)$ in the second phase. This trajectory, which has the property of ending at the desired distillate purity at final time, is chosen to be linear, with $x_{D, \text { ref }}\left(t_{s}\right)=0.925$ and $x_{D, r e f}\left(t_{f}\right)=0.9$. The accumulated distillate purity $x_{D}(t)$ is measured in Phase 2 .

In order to obtain a realistic test scenario, the following uncertainty is considered: 
- Perturbation: The vapor rate fluctuates every $0.5 h$ following an uniform distribution in the range $V=[13,17] \mathrm{kmol} / \mathrm{h}$

- Measurement noise: $5 \%$ multiplicative Gaussian noise is added to the product composition $x_{D}(t)$.

The values of the squared tracking error $\sum_{t_{s}}^{t_{f}} e^{2}(t)$ and the final tracking error $e\left(t_{f}\right)$ upon convergence are averaged over 20 realizations of the perturbation and measurement noise. Also, the variance $v_{e}\left(t_{f}\right)$ of the final tracking error is calculated from 20 realizations.

\subsubsection{Trajectory tracking via ILC}

Trajectory tracking involving a single output, $x_{D}(t)$, and a single input, $r(t)$, is implemented on a run-to-run basis via ILC. The initial input trajectory used for ILC is linear with $r\left(t_{s}\right)=0.898$ and $r\left(t_{f}\right)=0.877$. The residual tracking error cannot be reduced to zero for all times because of the non-zero tracking error at time $t_{s}$ arising from uncertainties in the start-up phase, that is, $x_{D}\left(t_{s}\right) \neq x_{D, r e f}\left(t_{s}\right)$. As a consequence, the ILC schemes that try to enforce zero residual tracking error do not converge in this case. Instead, non-zero tracking error has to be tolerated to enforce convergence. This can be accomplished by applying a forgetting factor or a time shift to the feedforward trajectory. Three ILC schemes without current-cycle feedback are considered, with $N=100$ time samples:

- $\underline{\beta=0.999}$. A forgetting factor $\beta$ is applied to the feedforward input trajectory in Eq. (26) with $\bar{A}=\beta I$ and $\bar{B}=K$.

- $\underline{\delta_{u}=0.25 h}$. A small shift is applied to the feedforward input trajectory in Eq. (29).

- $\delta_{u}=1 h, \delta_{e}=1 h$. The same large shift is applied to the feedforward input and the error trajectory in Eq. (29).

The proportional gain $K=0.1 I$ is determined as a compromise between robustness and performance. ILC with forgetting factor converges after 30 runs, while the schemes with time shift of the trajectories converge after 25 runs as illustrated in Figure 13. The final tracking error is slightly smaller with the 
latter methods, especially when the time shift of the feedforward trajectory is reduced to $\delta_{u}=0.25 h$ as shown in Table 3. Figure 14 shows the tracking performance of ILC with input shift after 20 runs. Note that, since it is necessary to have distillate in the product tank to be able to take measurements, tracking starts one sampling time after the start of Phase 2, that is, at at $t_{s}+h$.

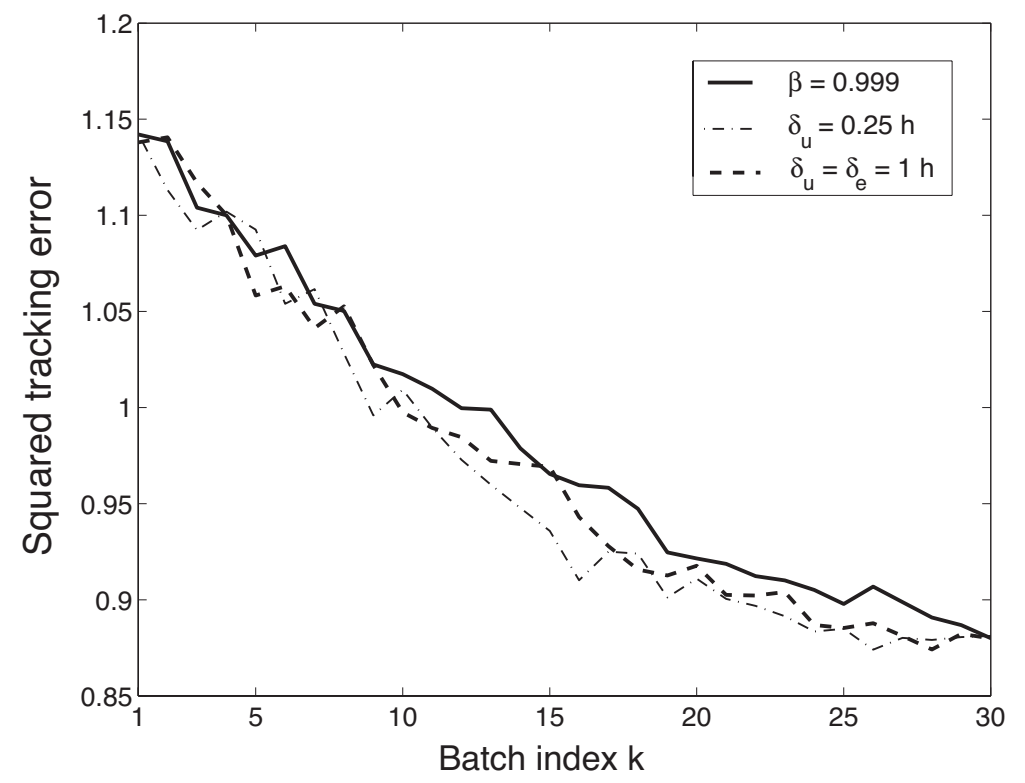

Figure 13: Evolution of the squared tracking error for three ILC schemes.

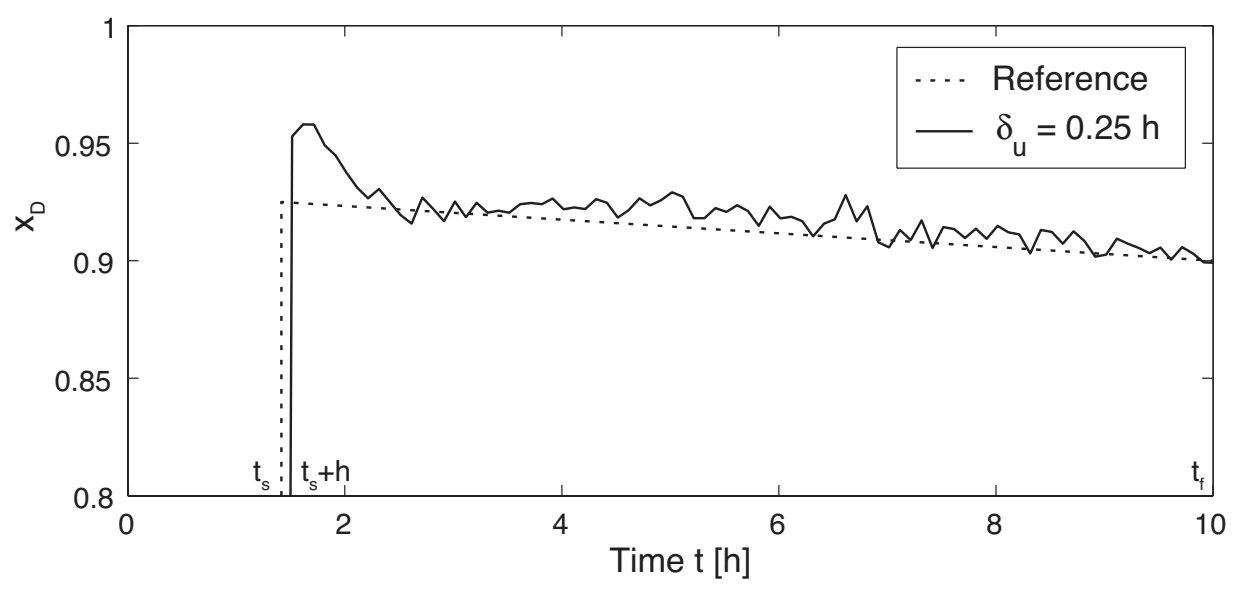

Figure 14: Tracking performance using ILC with input shift. 
Table 3: Comparison of the three tracking schemes after 30 runs in terms of the squared tracking error $\sum_{t_{s}}^{t_{f}} e^{2}(t)$, the tracking error at final time $e\left(t_{f}\right)$, and its variance $v_{e}\left(t_{f}\right)$.

\begin{tabular}{lccc}
\hline Strategy & $\sum_{t_{s}}^{t_{f}} e^{2}(t)$ & $\left|e\left(t_{f}\right)\right| \times 10^{3}$ & $v_{e}\left(t_{f}\right) \times 10^{5}$ \\
\hline \hline$\beta=0.999$ & 0.8738 & 6.5 & 4.1 \\
$\delta_{u}=0.25 h$ & 0.8745 & $\mathbf{0 . 5}$ & 3.0 \\
$\delta_{u}=1 h, \delta_{e}=1 h$ & 0.8833 & 2.2 & 3.5 \\
\hline
\end{tabular}




\section{PART B. OPTIMIZATION}

Process optimization is the method of choice for improving the performance of chemical processes while enforcing operational constraints ${ }^{63}$. Long considered as an appealing tool but only applicable to academic problems, optimization has now become a viable technology ${ }^{64,65}$. Still, one of the strengths of optimization, namely, its inherent mathematical rigor, can also be perceived as a weakness, since engineers might sometimes find it difficult to obtain an appropriate mathematical formulation to solve their practical problems. Furthermore, even when process models are available, the presence of plant-model mismatch and process disturbances makes the direct use of model-based optimal inputs hazardous.

In the last 30 years, the field of real-time optimization (RTO) has emerged to help overcome the aforementioned modeling difficulties. RTO integrates process measurements into the optimization framework. This way, process optimization does not rely exclusively on a (possibly inaccurate) process model but also on process information stemming from measurements. The first widely available RTO approach was the twostep approach that adapts the model parameters on the basis of differences between predicted and measured outputs and uses the updated process model to re-compute the optimal inputs ${ }^{66,67}$. However, in the presence of structural plant-model mismatch, this method is very unlikely to drive the plant to optimality ${ }^{68,69}$. Hence, alternatives to the two-step approach have recently been developed. For example, the modifier-adaptation scheme also proposes to solve a model-based optimization problem, but using a plant model with fixed model parameters ${ }^{69}$. Correction for uncertainty is made via modifier terms that are added to the cost and constraint functions of the optimization problem. As the modifiers include information on the differences between the predicted and the plant necessary conditions of optimality (NCO), this approach is prone to reach the plant optimum upon convergence. Yet another field has emerged, for which numerical optimization is not used online. With the so-called self-optimizing approaches ${ }^{70-72}$, the optimization problem is recast as a control problem that uses measurements to enforce certain optimality features for the plant. However, note that self-optimizing control ${ }^{70}$ and extremum-seeking control ${ }^{71}$, which have been developed to optimize continuous plants, are not suited to the optimization of batch processes. 
The optimization of a batch process falls naturally in the category of dynamic optimization, that is, with infinite-dimensional time profiles as decision variables. However, the introduction of input parameterization can transform the dynamic optimization problem into a static optimization problem with a countable number of decision variables, a so-called nonlinear program (NLP).

This optimization part is organized as follows. Various aspects of numerical optimization for both static and dynamic optimizations are presented first, followed by a discussion of two RTO classes, namely, repeated numerical optimization and optimizing control. The theoretical developments will then be illustrated by three case studies.

\section{Numerical Optimization}

Apart from very specific cases, the standard way of solving an optimization problem is via numerical optimization, for which a model of the process is required. As already mentioned, the optimization of a batch process can be formulated as a dynamic optimization problem. In this chapter, we will consider the following constrained dynamic optimization problem:

$$
\begin{aligned}
\min _{u\left[0, t_{f}\right]} & J:=\phi\left(x\left(t_{f}\right)\right) \\
\text { s.t. } & \dot{x}(t)=F(x(t), u(t)), \quad x(0)=x_{0}, \\
& S(x(t), u(t)) \leq 0 \\
& T\left(x\left(t_{f}\right)\right) \leq 0
\end{aligned}
$$

where $\phi$ is the terminal-time cost functional to be minimized, $x(t)$ the $n$-dimensional vector of state profiles with the known initial conditions $x_{0}, u(t)$ the m-dimensional vector of input profiles, $S$ the $n_{S}$-dimensional vector of path constraints that hold during the interval $\left[0, t_{f}\right], T$ the $n_{T}$-dimensional vector of terminal constraints that hold at $t_{f}$, and $t_{f}$ the final time, which can be either free or fixed ${ }^{\dagger}$. The optimization

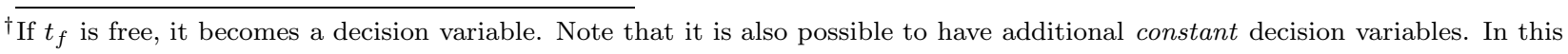
case, the decision variables would include the vector of input profiles $u\left[0, t_{f}\right]$ and the vector of constant quantities, $\rho$. This general case will not be considered here for simplicity of exposition, see ${ }^{72}$ for details. 
problem (57)-(60) is said to be in the Mayer form, that is, $J$ is a terminal-time cost. When an integral cost is added to $\phi$, the corresponding problem is said to be in the Bolza form, while when it only incorporates the integral cost, it is referred to as being in the Lagrange form. Note that these three formulations can be made equivalent by the introduction of additional states ${ }^{73}$.

The nonlinear dynamic optimization problem (57)-(60) is difficult to solve due to the time dependency of the various variables. In particular, the inputs $u(t)$ represent an infinite-dimensional decision vector. This time dependency can be removed via time discretization using, for example, the method of orthogonal collocation $^{74}$, which however results in a large vector of decision variables. Another approach consists in viewing the batch process as a static map between a small number of input parameters $\pi$ and a small number of run-end outputs $z$ as discussed in Section 3.3. These approximations reduce the dynamic optimization problem into a static problem. Next, the following problems will be addressed successively: dynamic optimization, reformulation of a dynamic optimization problem as a static optimization problem, static optimization, and effect of uncertainty.

\subsection{Dynamic Optimization}

Consider the optimization problem (57)-(60), for which we will present the necessary conditions of optimality.

\subsubsection{Pontryagin's minimum principle}

The NCO for a dynamic optimization problem are given by Pontryagin's minimum principle (PMP). The application of PMP provides insight in the optimal solution and generates explicit conditions for meeting active path and terminal constraints and forcing certain sensitivities to zero.

Let us define the Hamiltonian function $H(t)$,

$$
H(t)=\lambda^{T}(t) F(x(t), u(t))+\mu^{T}(t) S(x(t), u(t))
$$


and the augmented terminal cost

$$
\Phi\left(t_{f}\right)=\phi\left(x\left(t_{f}\right)\right)+\nu^{T} T\left(x\left(t_{f}\right)\right)
$$

where $\lambda^{T}(t)$ are the adjoint variables such that

$$
\dot{\lambda}^{T}(t)=-\frac{\partial H}{\partial x}(t), \quad \lambda^{T}\left(t_{f}\right)=\frac{\partial \Phi}{\partial x\left(t_{f}\right)},
$$

$\mu(t) \geq 0$ are the Lagrange multipliers associated with the path constraints, and $\nu \geq 0$ are the Lagrange multipliers associated with the terminal constraints. In dynamic optimization problems, there are both path and terminal objectives to satisfy, with these objectives being either constraints or sensitivities. PMP and the introduction of the Hamiltonian function $H(t)$ allow (i) assigning the NCO separately to path and terminal objectives, and (ii) expressing them in terms of meeting constraints and sensitivities, as illustrated in Table 4. The solution is generally discontinuous and consists of several intervals or arcs ${ }^{73,75}$. Each arc is characterized by a different set of active path constraints, that is, this set changes between successive arcs.

Table 4: NCO for a dynamic optimization problem ${ }^{73}$.

\begin{tabular}{ccc}
\hline \hline & Path & Terminal \\
Constraints & $\mu^{T}(t) S(x(t), u(t))=0$, & $\mu(t) \geq 0$ \\
Sensitivities & $\frac{\partial H}{\partial u}(t)=0$ & $\nu^{T} T\left(x\left(t_{f}\right)\right)=0, \quad \nu \geq 0$ \\
\hline
\end{tabular}

\subsubsection{Solution methods}

Solving the dynamic optimization problem (57)-(60) corresponds to finding the best input profiles $u\left[0, t_{f}\right]$ such that the cost functional is minimized, while meeting both the path and terminal constraints. Since the decision variables $u\left[0, t_{f}\right]$ are infinite dimensional, the inputs need to be parameterized using a finite set of parameters to be able to use numerical techniques. These techniques can be classified in two main categories ¥If the decision variables include the constant vector $\rho$, there will be terminal sensitivity elements for the determination of $\rho$, see $^{72}$ for details. 
according to the underlying formulation ${ }^{73}$, namely, direct optimization methods that solve the optimization problem (57)-(60) directly, and PMP-based methods that attempt to satisfy the NCO given in Table 4.

Direct optimization methods are distinguished further depending on whether the system equations are integrated explicitly or not ${ }^{73}$. In the sequential approach, the system equations are integrated explicitly, and the optimization is carried out in the space of the decision variables only. This corresponds to a "feasible-path" approach since the differential equations are satisfied at each step of the optimization. A piecewise-constant or piecewise-polynomial approximation of the inputs is often used. The computationally expensive part of the sequential approach is the accurate integration of the system equations, which needs to be performed even when the decision variables are far from the optimal solution!

In the simultaneous approach, an approximation of the system equations is introduced to avoid explicit integration for each candidate set of input profiles, thereby reducing the computational burden. Since the optimization is carried out in the full space of discretized inputs and states, the differential equations are satisfied only at the solution ${ }^{74,76}$. This is therefore called an "infeasible-path" approach. The direct approaches are by far the most commonly used. However, note that the input parameterization is often chosen arbitrarily by the user, which can affect both the efficiency and the accuracy of the approach ${ }^{77}$.

On the other hand, PMP-based methods try to satisfy the first-order NCO given in Table 4 . The NCO involve the state and adjoint variables, which need to be computed via integration. The differential equation system is a two-point boundary-value problem since initial conditions are available for the states and terminal conditions for the adjoints. The optimal inputs can be expressed analytically from the NCO in terms of the states and the adjoints, that is, $u^{*}\left[0, t_{f}\right]=\mathbf{U}\left(x\left[0, t_{f}\right], \lambda\left[0, t_{f}\right]\right)$. The resulting differentialalgebraic system of equations can be solved using a shooting approach ${ }^{78}$, that is, the decision variables include the initial conditions $\lambda(0)$ that are chosen in order to satisfy $\lambda\left(t_{f}\right)$. 


\subsection{Reformulation of a dynamic optimization problem as a static optimization problem}

Consider the optimization problem (57)-(60). Numerically, this problem can be solved by control vector parameterization $(\mathrm{CVP})^{76}$, whereby the infinite-dimensional inputs $u(t)$ are parameterized using a finite number of parameters $\pi$. Typically, the inputs are divided into small intervals, and a polynomial approximation of the inputs is used within each interval. The input parameter vector $\pi$ can also include the switching times between the intervals. It follows that the inputs can be expressed as $u(t)=\mathcal{U}(\pi, t)$ and the optimization performed with respect to $\pi$, where $\pi$ is the $n_{\pi}$-dimensional vector of constant decision variables.

The difficulty with such a parameterization is that the path constraints $S(x, u)$ have to be discretized as well, resulting in as many constraints as there are discretization points (typically a large number). However, if structural information regarding the shape of the optimal solution is available, the path constraints can be handled differently using an alternative parameterization as explained next.

The solution to a constrained terminal-time dynamic optimization problem is typically discontinuous and consists of various arcs. For a given input, the $i^{t h} \operatorname{arc} \eta_{i}(t)$ can be of two types:

1. $\eta_{i, p a t h}: \operatorname{Arc} \eta_{i}(t)$ is determined by an active path constraint (constraint-seeking arc),

2. $\eta_{i, \text { sens }}$ : Arc $\eta_{i}(t)$ is not governed by an active path constraint, it is sensitivity seeking.

Let us assume that the path constraints are met using either (i) assignment of input values in the case of active input bounds, or (ii) online feedback control via the measurement or estimation of the constrained quantities and their regulation to track the constraint values. The other parts of the inputs are parameterized as in CVP as $u(t)=\mathcal{U}(\pi, t)$. Since the path constraints are handled directly, they do not involve the decision variables $\pi$. Note that the knowledge of the active path constraints in various intervals is crucial for this parameterization. The final states, which affect the objective function and the terminal constraints, can be expressed in terms of the decision variables $\pi$ as follows:

$$
x\left(t_{f}\right)=x_{0}+\int_{0}^{t_{f}} F(x(t), \mathcal{U}(\pi, t)) d t:=\mathcal{F}(\pi),
$$


as $t_{f}$ is an element of $\pi$. Hence, the dynamic optimization problem (57)-(60) can be reformulated as:

$$
\begin{array}{ll}
\min _{\pi} & \phi(\mathcal{F}(\pi)) \\
\text { s.t. } & T(\mathcal{F}(\pi)) \leq 0,
\end{array}
$$

since the system dynamics are included in $\mathcal{F}$ and the path constraints are taken care of as mentioned above. It follows that the dynamic optimization problem can be reformulated as the following static NLP:

$$
\begin{array}{ll}
\min _{\pi} & J:=\Phi(\pi) \\
\text { s.t. } & G(\pi) \leq 0 .
\end{array}
$$

The scalar cost function to be minimized is written $\Phi(\pi):=\phi(\mathcal{F}(\pi))$, and the $n_{g}$-dimensional vector of constraints is written generically $G(\pi):=T(\mathcal{F}(\pi))$.

Some remarks are necessary at this point:

- This reformulation is a mathematical way of viewing the dynamic process as a static input-output " $\pi-(\Phi, G) "$ map. However, the process remains dynamic, with the terminal cost and the terminal constraints being affected by the process dynamics.

- This reformulation is clearly an approximation since it relies on: (i) a parameterization of the inputs, and (ii) approximate tracking of the state constraints.

- NLP (67)-(68) is a complex problem if one wants to predict $\Phi$ and $G$ from $\pi$ since the system dynamics need to be integrated according to Eq. (64). However, the situation is much simpler in the experimental context, as one simply apply the inputs $\pi$ to the plant and measure both $\Phi$ and $G$. In this case, the model used to predict $\Phi$ and $G$ is replaced by the plant itself. This is a perfect example of the power of measurement-based optimization compared to numerical optimization, as will be detailed later.

\subsection{Static Optimization}

The optimization problem is of algebraic nature with a finite number of constant decision variables. 


\subsubsection{KKT necessary conditions of optimality}

With the formulation (67)-(68) and the assumption that the cost function $\Phi$ and the constraint functions $G$ are differentiable, the Karush-Kuhn-Tucker (KKT) conditions read ${ }^{79}$ :

$$
\begin{aligned}
G\left(\pi^{*}\right) & \leq 0, \\
\nabla \Phi\left(\pi^{*}\right)+\left(\nu^{*}\right)^{T} \nabla G\left(\pi^{*}\right) & =0, \\
\nu^{*} & \geq 0, \\
\left(\nu^{*}\right)^{T} G\left(\pi^{*}\right) & =0,
\end{aligned}
$$

where $\pi^{*}$ denotes the solution, $\nu^{*}$ the $n_{g}$-dimensional vector of Lagrange multipliers associated with the

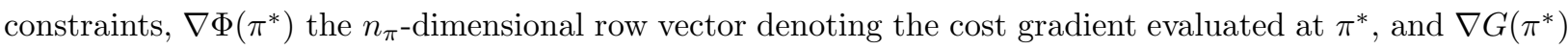
the $\left(n_{g} \times n_{\pi}\right)$-dimensional Jacobian matrix computed at $\pi^{*}$. For these equations to be necessary conditions, $\pi^{*}$ needs to be a regular point for the constraints, which calls for linear independence of the active constraints, that is, $\operatorname{rank}\left\{\nabla G_{a}\left(\pi^{*}\right)\right\}=n_{g, a}$, where $G_{a}$ represents the set of active constraints, whose cardinality is $n_{g, a}$.

Condition (69) is the primal feasibility condition, Condition (71) the dual feasibility condition, and Condition (72) the complementarity slackness condition. The stationarity condition (70) indicates that, at the solution, collinearity between the cost gradient and a linear combination of the gradients of the active constraints prevents from finding a search direction that would result in cost reduction while still keeping the constraints satisfied.

\subsubsection{Solution methods}

Static optimization can be solved by state-of-the-art nonlinear programming techniques. In the presence of constraints, the three most popular approaches are ${ }^{80}$ : (i) penalty-function methods, (ii) interior-point methods, and (iii) sequential quadratic programming. The main idea in penalty-function methods is to replace the solution to a constrained optimization problem by the solution to a sequence of unconstrained optimization problems. This is made possible by incorporating the constraints in the objective function via 
a penalty term, which penalizes any violation of the constraints, while guaranteeing that the two problems share the same solution (by selecting sufficiently large weights). Interior-point methods also incorporate the constraints in the objective function ${ }^{81}$. However, the constraints are approached from the feasible region, and the additive terms increase to become infinitely large at the value of the constraints, thereby acting more like a barrier than a penalty term. A clear advantage of interior-point methods is that feasible iterates are generated, while for penalty function methods, feasibility is only guaranteed upon convergence. Note that a barrier-penalty function that combines the advantages of both approaches has also been proposed ${ }^{82}$. Another way of computing the solution to a static optimization problem is to solve the set of NCO, for example iteratively using sequential quadratic programming (SQP). SQP methods solve a sequence of optimization sub-problems, each one minimizing a quadratic approximation to the Lagrangian function $L:=\Phi+\nu^{T} G$ subject to a linear approximation of the constraints. SQP typically uses Newton's or quasi-Newton methods to solve the KKT conditions.

\subsection{Effect of Uncertainty}

\subsubsection{Plant-model mismatch}

The model used for optimization consists of a set of equations that represent an abstract view, yet always a simplification, of the real process. Such a model is built based on conservations laws (for mass, numbers of moles, energy) and constitutive relationships that express kinetics, equilibria and transport phenomena. The simplifications that are introduced at the modeling stage to obtain a tractable model affect the quality of the process model in two ways:

(i) some physical or chemical phenomena are assumed to be negligible and are discarded, and

(ii) some dynamic equations are assumed to be at quasi-steady state.

Hence, the structure of the model that is used differs from the "true" model structure. This dichotomy gives rise to the so-called structural plant-model mismatch. Furthermore, the model involves a 
number of physical parameters, whose values are not known accurately. These parameters are identified using process measurements and, consequently, are only known to belong to some interval with a certain probability. For the sake of simplicity, we will consider thereafter that all modeling uncertainties, although unknown, can be incorporated in the vector of uncertain parameters $\theta$.

\subsubsection{Model adequacy}

Uncertainty is detrimental to the quality of both model predictions and optimal solutions. If the model is not able to predict the process outputs accurately, it will most likely not be able to predict the NCO correctly. On the other hand, even if the model is able to predict the process outputs accurately, it is often unable to predict the NCO correctly since it has been trained to predict the outputs and not, for instance, the gradients that are key constituents of the NCO. Hence, numerical optimization is capable of computing optimal inputs for the model, but it often fails to reach plant optimality.

The property that ensures that a model-based optimization problem will be able to determine the optimal inputs for the plant is referred to in the literature as "model adequacy". For a given model-based RTO scheme, a model is adequate if the RTO scheme is able to predict the correct set of active plant constraints and the correct alignment of plant gradients. Model adequacy represents a major challenge in process optimization since, as discussed earlier, models are trained to predict the plant outputs rather than the plant NCO. In practice, application of model-based optimal inputs leads to suboptimal, and often infeasible, operation.

\section{Real-time Optimization}

In the presence of modeling errors and process disturbances, the control trajectories computed offline lose their optimal character. One way to reject the effect of uncertainty on the overall performance (with respect to both optimality and feasibility) is by adequately incorporating process measurements in the optimization 
framework. This is the field of real-time optimization. Measurements can be incorporated in two different ways as illustrated in Figure 15:

a. Adapt the process model and repeat the optimization. At each iteration, the model parameters are updated and the optimization problem solved numerically to generate $u^{*}\left[0, t_{f}\right]$. This adaptation and optimization can also be repeated online, in a single run, to estimate at time $t$ the current states $\hat{x}(t)$ and compute the inputs $u^{*}\left[t, t_{f}\right]$ for the remaining part of the batch.

b. Adapt the inputs through optimizing feedback control. Here, the optimization is implicit since optimality and feasibility are enforced via feedback control to satisfy the necessary conditions of optimality. The control scheme often involves (i) online elements that generate parts of the inputs, $u_{a}^{*}(t)$, and (ii) run-to-run elements that generate the other part of the inputs, $u_{b}^{*}\left[0, t_{f}\right]$, via the input parameters $\pi^{*}$.

These RTO schemes will be discussed in the following subsections.

\subsection{Repeated Numerical Optimization}

As additional information about the process becomes available, either during or at the end of the run, this information can be used to improve future operations. This will be documented next for both online implementation using economic MPC and run-to-run implementation via the two-step approach.

\subsubsection{Economic MPC (Strategy 1)}

The approach is similar to MPC discussed in Section 4.2.1, but for the fact that the cost function is no longer tailored to tracking run-end references, but rather to minimize an economic cost function. The problem can 


\begin{tabular}{|c|c|c|}
\hline \multirow{2}{*}{$\begin{array}{l}\text { Implementation } \\
\text { aspect }\end{array}$} & \multicolumn{2}{|c|}{ Optimization via } \\
\hline & $\begin{array}{l}\text { Repeated num. optim. } \\
\text { (of an updated model) }\end{array}$ & $\begin{array}{c}\text { Optimizing feedback control } \\
\text { (track optimality conditions) }\end{array}$ \\
\hline $\begin{array}{l}\text { Online } \\
u^{*}(t), u^{*}\left[t, t_{f}\right]\end{array}$ & $\begin{array}{l}\text { (1) Economic MPC } \\
u^{*}\left[t, t_{f}\right] \rightarrow \mathrm{P} \rightarrow y(t) \rightarrow \mathrm{E} \rightarrow \hat{x}(t) \\
\text { eMPC }\end{array}$ & $\begin{array}{r}\text { 3a Online NCO tracking } \\
\qquad \begin{array}{c}u_{a}^{*}(t) \longrightarrow \mathrm{P} \longrightarrow y(t) \\
\square \text { track path objectives }\end{array}\end{array}$ \\
\hline $\begin{array}{c}\text { Run-to-run } \\
u^{*}\left[0, t_{f}\right]\end{array}$ & $\begin{array}{l}2 \text { Two-step approach } \\
u^{*}\left[0, t_{f}\right] \longrightarrow \mathrm{P} \longrightarrow y\left[0, t_{f}\right] \\
\text { Id. \& Opt. for next run }\end{array}$ & $\begin{array}{l}\text { 3b Run-to-run NCO tracking } \\
u_{b}^{*}\left[0, t_{f}\right] \longrightarrow \mathrm{P} \longrightarrow z \\
\Delta \begin{array}{c}\text { track terminal objectives } \\
\text { on a run-to run basis }\end{array}\end{array}$ \\
\hline
\end{tabular}

Figure 15: RTO schemes for batch processes. The schemes are classified according to whether the optimization is implemented via repeated numerical optimization or feedback control (horizontal division) and whether it is implemented online or on a run-to-run basis (vertical division). eMPC stands for "economic model predictive control". Note that NCO tracking can be implemented using the four control approaches given in Figure 2 to track the path and terminal objectives either online or on a run-to-run basis. Most often, the path constraints are implemented online, while the terminal objectives are implemented on a run-to-run manner (see the similarities with Table 4). 
be formulated as follows:

$$
\begin{array}{cl}
\min _{u\left[t_{i}, t_{f}\right]} & J:=\phi\left(x\left(t_{f}\right)\right) \\
\text { s.t. } & \dot{x}(t)=F(x(t), u(t)), \quad x\left(t_{i}\right)=\hat{x}\left(t_{i}\right), \\
& S(x(t), u(t)) \leq 0, \\
& T\left(x\left(t_{f}\right)\right) \leq 0, \\
& x\left(t_{f}\right) \in \mathcal{X},
\end{array}
$$

where $t_{i}$ is the discrete time instant at which the optimization is performed, $\hat{x}\left(t_{i}\right)$ is the state estimate at that time, and $\mathcal{X}$ is the bounded region of state space where the final state should lie. Numerical optimization yields the control sequence, $u^{*}\left[t_{i}, t_{f}\right]$, of which only the first part, $u^{*}\left[t_{i}, t_{i}+h\right]$, is applied in an open-loop fashion to the plant. Numerical optimization is then repeated at every sampling instant. The scheme is illustrated in Figure 16.

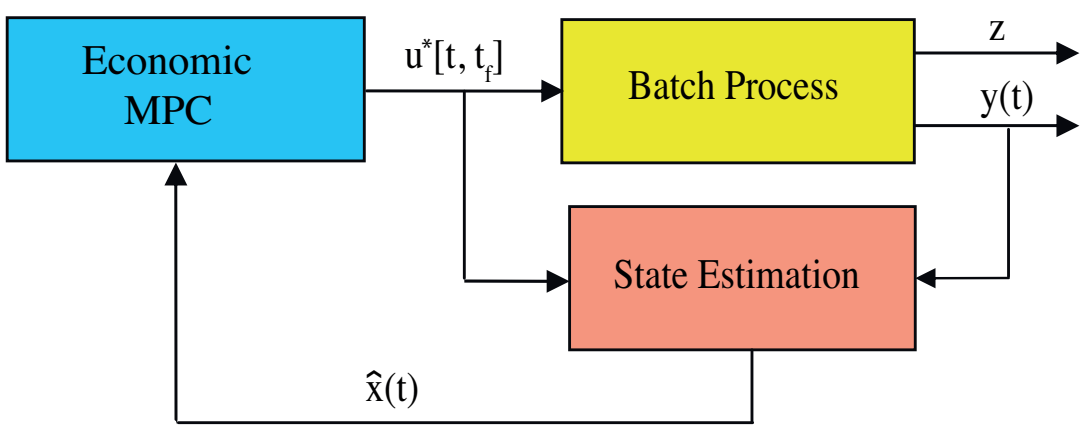

Figure 16: Economic MPC to optimize a batch process repeatedly online.

Note that re-optimization performed during a run requires a valid process model, including estimates of the current states. Similarly, correction through state feedback requires knowledge of the current states or their estimates. Hence, the main engineering challenge in the context of batch processes lies in estimating the states and the parameters from a few noisy measurements. Although the use of extended Kalman filters ${ }^{83}$ or moving-horizon estimation ${ }^{84}$ has become increasingly common and successful for continuous processes, these methods are difficult to use for batch processes due to the large variations that the states typically go through and the limited time of operation. Good accounts of these difficulties are available ${ }^{85,86}$. 
The weakness of this method is clearly its reliance on the model; if the model parameters are not updated, model accuracy plays a crucial role. However, if the model is updated, there is a conflict between parameter estimation and optimization, the so-called dual control problem ${ }^{87}$, since parameter estimation requires persistency of excitation, that is, the inputs must be sufficiently varied to uncover the unknown parameters, a condition that is usually not satisfied when near-optimal inputs are applied. Finally, note that the scheme is called "economic MPC" because MPC typically addresses the tracking of given trajectories. Here, there are no optimal trajectories to track, but rather some economic cost function to minimize. This topic has gained a lot of interest in recent years ${ }^{88}$.

\subsubsection{Two-step approach (Strategy 2)}

In the two-step approach, measurements are used to refine the model, which is then used to optimize the process ${ }^{67}$. The two-step approach has gained popularity over the past thirty years mainly because of its conceptual simplicity. Yet, the two-step approach is characterized by certain intrinsic difficulties that are often overlooked. In its iterative version, the two-step approach involves two optimization problems, namely, one each for parameter identification and process optimization as shown in Figure 17 and described next:

$$
\begin{aligned}
& \text { Identification } \quad \hat{\theta}_{k}:=\arg \min _{\theta}\left(\left\|y_{p, k}\left[0, t_{f}\right]-y_{k}\left[0, t_{f}\right]\right\|_{l_{2}}\right) \\
& \text { s.t. } \quad \dot{x}_{k}(t)=F\left(x_{k}(t), u_{k}^{*}(t), \theta\right), \quad x_{k}(0)=x_{0, k}, \\
& y_{k}(t)=H\left(x_{k}(t), u_{k}^{*}(t), \theta\right), \\
& \theta \in \Theta \\
& \text { Optimization } \quad u_{k+1}^{*}\left[0, t_{f}\right]:=\arg \min _{u\left[0, t_{f}\right]} \phi\left(x_{k}\left(t_{f}\right)\right) \\
& \text { s.t. } \quad \dot{x}_{k}(t)=F\left(x_{k}(t), u(t), \hat{\theta}_{k}\right), \quad x(0)=x_{0, k}, \\
& S\left(x_{k}(t), u(t), \hat{\theta}_{k}\right) \leq 0 \\
& T\left(x_{k}\left(t_{f}\right), \hat{\theta}_{k}\right) \leq 0
\end{aligned}
$$

where $\Theta$ indicates the set in which the uncertain parameters $\theta$ are assumed to lie. The subscript $(\cdot)_{p}$ is used to indicate that the outputs are the plant measurements $y_{p, k}\left[0, t_{f}\right]$, by opposition to the values predicted by the model, $y_{k}\left[0, t_{f}\right]$. 


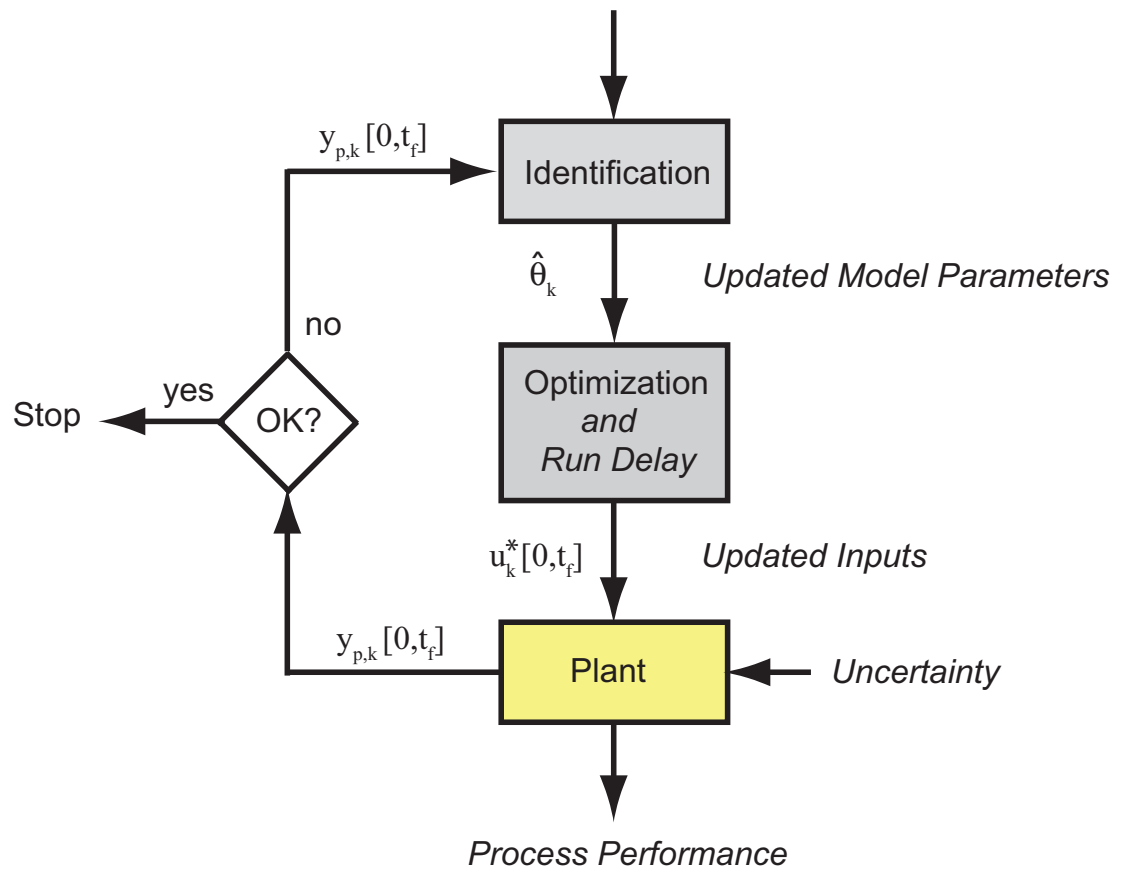

Figure 17: Basic idea of the two-step approach with its two optimization problems.

The first step identifies best values for the uncertain parameters by minimizing the $l_{2}$-norm of the output prediction errors. The updated model is then used for computing the optimal inputs for the next iteration. Algorithmically, the optimization of the performance of a batch process proceeds as follows:

1. Initialization. Set $k=1$ and select an initial guess for the input profiles $u_{k}^{*}\left[0, t_{f}\right]$.

2. Iteration. Apply the inputs $u_{k}^{*}\left[0, t_{f}\right]$ to the plant. Complete the batch while measuring the run-time outputs $y_{p, k}\left[0, t_{f}\right]$.

3. Compute the distance between the predicted and measured outputs and continue if this distance exceeds the tolerance, otherwise stop.

4. Solve the identification problem (P1) and compute $\hat{\theta}_{k}$.

5. Solve the optimization problem (P2) and compute $u_{k+1}^{*}\left[0, t_{f}\right]$. Set $k:=k+1$ and repeat Steps 2-5.

The two-step approach suffers from two main limitations. First, the identification problem requires sufficient excitation, which is however rarely the case since the inputs are computed for optimality rather 
than for the sake of identification. Hence, one has to make sure that there is sufficient excitation, for example via a dual optimization approach that adds a constraint to the optimization problem regarding the accuracy of the estimated parameters ${ }^{89}$. The second limitation is inherent to the philosophy of the method. Since the adjustable handles are the model parameters, the method assumes that (i) all the uncertainty (including process disturbances) can be represented by the set of uncertain parameters, which is rarely the case.

\subsection{Optimizing Feedback Control}

The second class of RTO methods proposes to adapt the process inputs via feedback control, that is, without repeating the numerical optimization. Since feedback control is used to enforce the necessary conditions of optimality of the dynamic optimization problem, the resulting scheme is called "NCO tracking".

\subsubsection{NCO tracking (Strategy 3)}

NCO tracking is a feedback control scheme, where the controlled variables (CVs) correspond to measurements or estimates of the plant $\mathrm{NCO}$, and the manipulated variables (MVs) are appropriate elements of the input profiles. Enforcing the plant NCO is indeed an indirect way of solving the optimization problem for the plant, along the lines of the PMP-based methods discussed in Section 8.1.2.

Necessary conditions of optimality. The NCO of the dynamic optimization problem (57)-(60) encompass four parts as shown in Table 4: (i) the path constraints, (ii) the path sensitivities, (iii) the terminal constraints, and (iv) the terminal sensitivities. There are as many NCO as there are degrees of freedom in the optimization problem, thus making the system of equations perfectly determined. NCO tracking proposes to enforce the four NCO parts via tailored feedback control. In particular, some objectives are met using online control, while others are met via run-to-run control, thereby fully exploiting the versatility of control approaches for batch processes, as discussed in Sections 4 and 5. The NCO-tracking scheme is therefore a two-level (online and run-to-run) multivariable feedback control problem, as illustrated in Figure 18. The design of the NCO-tracking controller is supported by the concept of "solution model" 72 . 


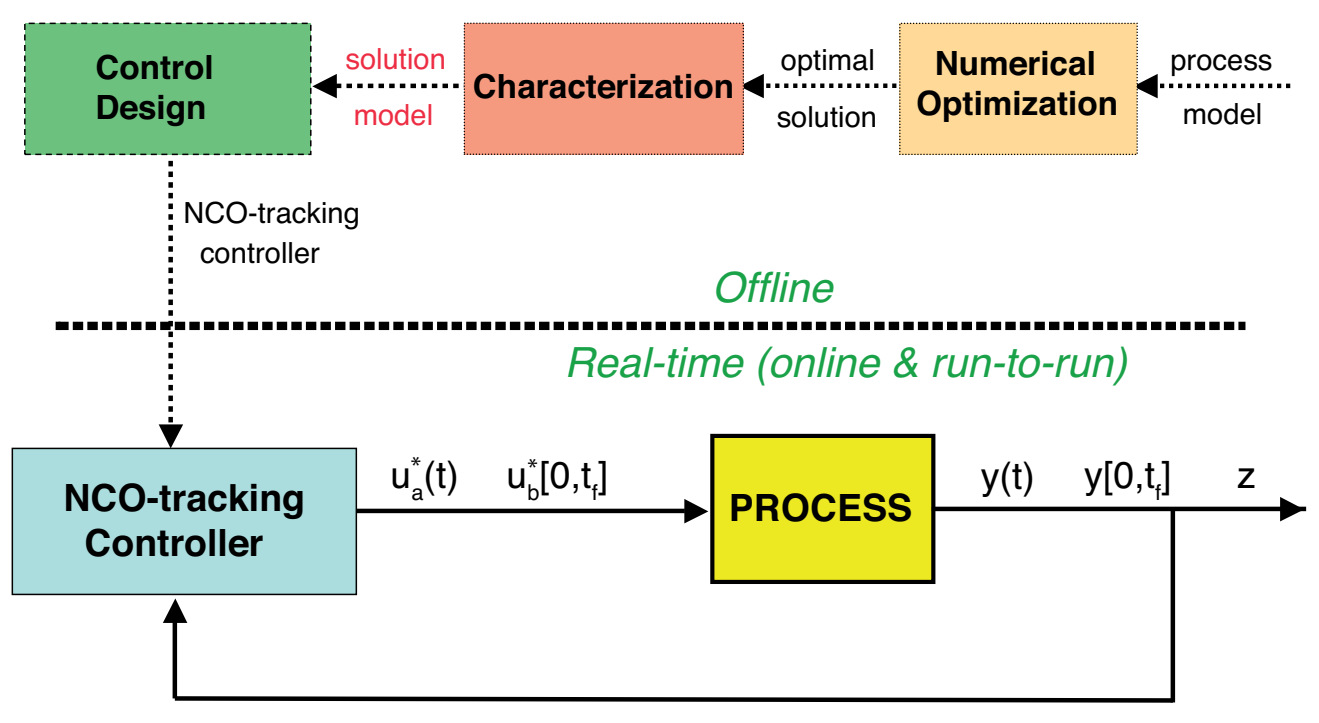

Figure 18: NCO-tracking scheme. The offline activities include the generation of a solution model and the design of a NCO-tracking controller. The real-time activities involve online and run-to-run control to enforce the plant NCO; $u_{a}^{*}(t)$ and $u_{b}^{*}\left[0, t_{f}\right]$ are the input parts that are generated online and on a run-to-run basis, respectively.

Solution model. The solution model is a qualitative model of the optimal solution, which includes (i) the types and sequence of arcs, thus assuming no change in active constraints, (ii) the degrees of freedom (MVs) that one would like to use for control, and (iii) the corresponding NCO (CVs). It is important to understand that the solution model is simply a tool that helps solve the problem at hand in an efficient way. Although there may be an exact solution model for the process model at hand, this is certainly not the case for the unknown plant. Hence, the designer will be able to propose alternative (from simple to more complex) solution models to tackle the design of the multivariable control problem. This aspect, which represents one of the strengths of NCO tracking, will be detailed in the first case study.

The development of a solution model involves three main steps:

1. Characterize the optimal solution in terms of the types and sequence of arcs by performing numerical optimization using the best available plant model. This plant model need not be very accurate, but it ought to provide the correct types and sequence of arcs. One typically performs a robustness analysis 
to ensure that the qualitative solution remains structurally valid in presence of uncertainty.

2. Select a finite set of input arcs and parameters to represent (or approximate) the input profiles, and formulate the NCO for this choice of degrees of freedom. Note that the NCO will change with the choice of the degrees of freedom.

3. Pair the MVs and the NCO to form a multivariable control problem.

Steps 2 and 3 might require iterations since they greatly affect the implementation that is discussed next.

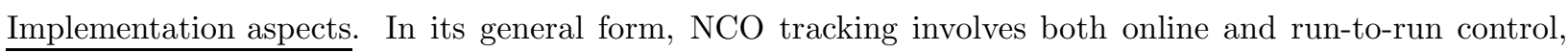
as determined by the solution model and the resulting MV-CV pairing. Some NCO elements are typically implemented online, while others are easier on a run-to-run basis. For example, a path constraint is easily enforced online via constraint control, while both terminal constraints and terminal sensitivities are easier to meet iteratively over several runs. The decision regarding which NCO elements to implement online and which on a run-to-run basis depends on the nature of the various arcs. For a given input, the various arcs can be of two types:

(i) A constraint-seeking input arc is associated with a path constraint being active in a given time interval. If the path constraint is an input bound, the input is simply set at the bound, while in the case of a state constraint, feedback control can be used to track the value of the constrained quantity.

(ii) A sensitivity-seeking input arc requires $\frac{\partial H}{\partial u}(t)=0$, which is difficult to implement as such since $H(t)$ is a function of the adjoint variables $\lambda(t)$. Hence, one tries to approximate the sensitivity-seeking arc using a parsimonious input parameterization with only a few constant parameters. This way, the number of degrees of freedom are no longer the infinite-dimensional $u(t)$, but rather the few constant input parameters. Furthermore, with the input parameterization, the batch process is viewed as the static map $z=M(\pi)$ given by Eq. (7). Consequently, the path condition $\frac{\partial H}{\partial u}(t)=0$ is replaced by terminal conditions of the type $\frac{\partial \phi\left(x\left(t_{f}\right)\right)}{\partial \pi}=0$, which can be enforced on a run-to-run basis. Note that the effect of the approximations introduced at the solution level can be assessed in terms of optimality loss. 
In summary, the ease of implementation and therefore the success of NCO tracking depends on the quality of the approximations that are introduced to generate the solution model (choice of MVs, corresponding $\mathrm{NCO}$, and pairing MV-NCO). These are true engineering decisions that are made with plant optimality in mind!

\section{Optimization Applications}

\subsection{Semi-batch Reactor with Safety and Selectivity Constraints}

A simple semi-batch reactor with jacket cooling is considered to illustrate the NCO-tracking approach and, in particular, the generation of alternative solution models ${ }^{72}$.

\subsubsection{Problem formulation}

- Reaction system: $A+B \rightarrow C, 2 B \rightarrow D$, isothermal, exothermic reactions.

- Objective: Maximize the amount of $C$ at a given final time.

- Manipulated input: Feedrate of $B$.

- Path constraints: Input bounds; heat-removal constraint expressed as a lower bound on the cooling jacket temperature.

- Terminal constraint: Upper bound on the amount of $D$ at final time. 
Model equations. Assuming perfect control of the reactor temperature through adjustment of the cooling jacket temperature, the model equations read:

$$
\begin{array}{rlr}
\dot{c_{A}} & =-k_{1} c_{A} c_{B}-\frac{u}{V} c_{A}, & c_{A}(0)=c_{A, 0}, \\
\dot{c_{B}} & =-k_{1} c_{A} c_{B}-2 k_{2} c_{B}^{2}+\frac{u}{V}\left(c_{B_{i n}}-c_{B}\right), & c_{B}(0)=c_{B, 0}, \\
\dot{V} & =u, & V(0)=V_{0}, \\
T_{j} & =T_{r}-\frac{V}{U A}\left[\left(-\Delta H_{1}\right) k_{1} c_{A} c_{B}+\left(-\Delta H_{2}\right) k_{2} c_{B}^{2}\right], \\
n_{C} & =n_{A, 0}-n_{A}, \\
n_{D} & =\frac{1}{2}\left[\left(n_{A}-n_{A, 0}\right)-\left(n_{B}-n_{B, 0}\right)+c_{B_{i n}}\left(V-V_{0}\right)\right],
\end{array}
$$

where the number of moles of Species $\mathrm{X}$ is defined as $n_{X}(t)=V(t) c_{X}(t)$.

$\underline{\text { Variables and parameters. }} c_{X}$ : concentrations of Species $X, n_{X}$ : number of moles of species $X, V:$ reactor volume, $k_{i}$ : kinetic coefficient of reaction $i, u$ : feedrate of $B, c_{B_{i n}}$ : inlet concentration of $B, \Delta H_{i}$ : enthalpy of reaction $i, T_{r}$ : reactor temperature, $T_{j}$ : cooling jacket temperature, $U$ : heat-transfer coefficient, $A$ : reactor heat-exchange area. The model, operating and optimization parameters are given in Table 5.

Table 5: Model parameters, operating bounds and initial conditions.

\begin{tabular}{|lll|lll|}
\hline$k_{1}$ & 0.11 & $\mathrm{~L} /(\operatorname{mol} \min )$ & $k_{2}$ & 0.13 & $\mathrm{~L} /(\mathrm{mol} \min )$ \\
$\Delta H_{1}$ & $-8 \times 10^{4}$ & $\mathrm{~J} / \mathrm{mol}$ & $\Delta H_{2}$ & $-10^{5}$ & $\mathrm{~J} / \mathrm{mol}$ \\
$U A$ & $1.25 \times 10^{4}$ & $\mathrm{~J} /\left(\min { }^{\circ} \mathrm{C}\right)$ & $c_{B_{i n}}$ & 5 & $\mathrm{~mol} / \mathrm{L}$ \\
$T_{r}$ & 30 & ${ }^{\circ} \mathrm{C}$ & $T_{j, \min }$ & 10 & ${ }^{\circ} \mathrm{C}$ \\
$u_{\max }$ & 1 & $\mathrm{~L} / \min$ & $n_{D, \max }$ & 100 & $\mathrm{~mol}$ \\
$c_{A, 0}$ & 0.5 & $\mathrm{~mol} / \mathrm{L}$ & $c_{B, 0}$ & 0 & $\mathrm{~mol} / \mathrm{L}$ \\
$V_{0}$ & 1000 & $\mathrm{~L}$ & $t_{f}$ & 180 & $\mathrm{~min}$ \\
\hline
\end{tabular}


Optimization problem. The objective of maximizing the amount of $C$ at the given final time $t_{f}$ can be written mathematically as follows:

$$
\begin{aligned}
\max _{u\left[0, t_{f}\right]} & n_{C}\left(t_{f}\right) \\
\text { s.t. } & \text { dynamic model }(78)-(83), \\
& 0 \leq u(t) \leq u_{\max }, \\
& T_{j}(t) \geq T_{j, \min }, \\
& n_{D}\left(t_{f}\right) \leq n_{D, \max } .
\end{aligned}
$$

\subsubsection{Characterization of the optimal solution}

The optimal feedrate profile depicted in Figure 19 exhibits three intervals:

- The feedrate is initially at its upper bound, $u_{\text {bound }}(t)=u_{\max }$, in order to attain the heat-removal constraint as quickly as possible.

- Then, $u_{\text {path }}(t)$ keeps the path constraint $T_{j}(t)=T_{j, \min }$ active.

- Finally, the input switches to $u_{\text {sens }}(t)$ to take advantage of the best compromise between producing the desired $C$ and the undesired $D$. The switching time $t_{2}$ is determined in such a way that the terminal constraint $n_{D}\left(t_{f}\right)=n_{D, \max }$ is met at final time.

Using PMP, it can be shown that the competition between the two reactions results in a sensitivityseeking feedrate reflecting the optimal compromise between producing $C$ and $D$. This sensitivity-seeking input can be calculated from the second time derivative of $H_{u}=0$ as:

$$
u_{\text {sens }}(t)=\frac{n_{B}\left(-k_{1} c_{A} c_{B}+2 k_{1} c_{A} c_{B i n}+4 k_{2} c_{B} c_{B i n}\right)}{2 c_{B i n}\left(c_{B i n}-c_{B}\right)} .
$$

On the other hand, the constraint-seeking $\operatorname{arc} u_{\text {path }}$ corresponds to riding along the path constraint $T_{j}(t)=$ $T_{j, m i n}$. The input is obtained by differentiating the path constraint once with respect to time, that is, from $\dot{T}_{j}=0$ :

$$
u_{p a t h}(t)=\frac{c_{B} V\left(\Delta H_{1} k_{1} c_{A}\left(k_{1} c_{B}+k_{1} c_{A}+2 k_{2} c_{B}\right)+2 \Delta H_{2} k_{2} c_{B}\left(k_{1} c_{A}+2 k_{2} c_{B}\right)\right)}{\Delta H_{1} k_{1} c_{A}\left(c_{B i n}-c_{B}\right)+\Delta H_{2} k_{2} c_{B}\left(2 c_{B i n}-c_{B}\right)} .
$$




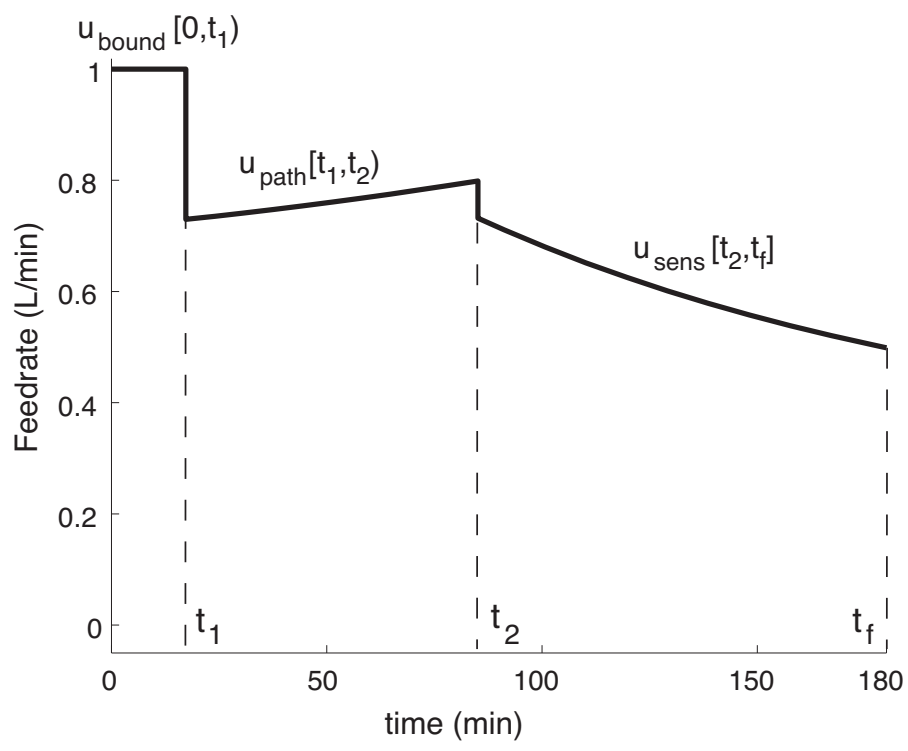

Figure 19: Optimal feedrate consisting of the three $\operatorname{arcs} u_{\text {bound }}\left[0, t_{1}\right), u_{\text {path }}\left[t_{1}, t_{2}\right)$ and $u_{\text {sens }}\left[t_{2}, t_{f}\right]$.

Since analytical expressions exist for all three input arcs, and $t_{1}$ can be determined upon $T_{j}(t)$ reaching $T_{j, m i n}$, the optimal solution can be parameterized using a single parameter, namely, the switching time $t_{2}$ between $u_{\text {path }}(t)$ and $u_{\text {sens }}(t)$. Note that the input arc $u_{\text {path }}(t)$ given by Eq. (89) will keep the reactor on the path constraint only for the case of a perfect model and perfect measurements. Fortunately, in the presence of uncertainty, one can implement $u_{p a t h}(t)$ by forcing $T_{j}(t)$ to track the reference $T_{j, \min }$, which is straightforward to implement using, for example, PI control. Similarly, the arc $u_{\text {sens }}(t)$ is only optimal for the case of a perfect model and perfect measurements. The way around this difficulty is best handled with the selection of an appropriate solution model.

\subsubsection{Alternative solution models}

A key feature of NCO tracking is the possibility to choose appropriate MVs and CVs and, if necessary, to introduce approximations. Hence, the choice of MVs and CVs is not made once for all, but it may require iterations. In fact, the appropriateness of these choices is often key to the success of NCO tracking in practice. This is illustrated next for this example, whereby three alternative solution models are proposed, all with the same objective but with very different implementation aspects. 
This solution model has the structure of the optimal solution given in Figure 19. The decision variables (MVs) associated with the feedrate input $u\left[0, t_{f}\right]$ are the three $\operatorname{arcs} u\left[0, t_{1}\right), u\left[t_{1}, t_{2}\right)$, and $u\left[t_{2}, t_{f}\right]$ and the two switching times $t_{1}$ and $t_{2}$. The corresponding $\mathrm{NCO}(\mathrm{CVs})$ include three path constraints and two pointwise constraints at times $t_{1}$ and $t_{f}$ (Table 6$)$.

Table 6: NCO for Solution model A (pointwise constraints are underlined).

\begin{tabular}{ccc}
\hline \hline & Path & Terminal \\
Constraints & $u\left[0, t_{1}\right)=u_{\max }$ & $\underline{n_{D}\left(t_{f}\right)=n_{D, \max }}$ \\
& $\frac{T_{j}\left(t_{1}\right)=T_{j, \min }}{}$ & \\
& $T_{j}\left[t_{1}, t_{2}\right)=T_{j, \min }$ & \\
Sensitivities & $\frac{\partial H}{\partial u}\left[t_{2}, t_{f}\right]=0$ & - \\
\hline
\end{tabular}

The pairing between MVs and CVs is rather straightforward:

1. $u\left[0, t_{1}\right)$ is implemented open loop as $u\left[0, t_{1}\right)=u_{\max }$.

2. $t_{1}$ is determined when $T_{j}(t)$ reaches $T_{j, \min }$.

3. $u\left[t_{1}, t_{2}\right)$ is determined by tracking the path constraint $T_{j}(t)=T_{j, m i n}$.

4. $t_{2}$ is determined from the terminal condition $n_{D}\left(t_{f}\right)=n_{D, \max }$.

5. And finally, $u\left[t_{2}, t_{f}\right]$ is determined form the path sensitivity $\frac{\partial H}{\partial u}\left[t_{2}, t_{f}\right]=0$.

If the pairing is easily formulated, it is rather difficult to implement this control strategy in practice. Steps 1-3 are straightforward. Step 4 requires prediction, which requires a model to be done online or, otherwise, could be implemented on a run-to-run basis. Step 5 requires a model and the computation of the Hamiltonian function $H(t)$ that includes the adjoint variables $\lambda(t)$. Hence, Solution model A is not very useful for implementation. 
We introduce the parameterization $u_{\text {sens }}\left[t_{2}, t_{f}\right]=\pi$, which allows approximating the last arc with a single parameter. The corresponding NCO include two path constraints and three pointwise constraints at times $t_{1}$ and $t_{f}$ (Table 7$)$.

Table 7: NCO for Solution model B (pointwise constraints are underlined).

\begin{tabular}{ccc}
\hline \hline Path & Terminal \\
Constraints & $u\left[0, t_{1}\right)=u_{\max }$ & $\frac{n_{D}\left(t_{f}\right)=n_{D, \text { max }}}{}$ \\
& $\frac{T_{j}\left(t_{1}\right)=T_{j, \min }}{}$ \\
& $T_{j}\left[t_{1}, t_{2}\right)=T_{j, \min }$ & \\
Sensitivities & - & $\frac{\frac{\partial n_{C}\left(t_{f}\right)}{\partial \pi}=0}{2}$ \\
\hline
\end{tabular}

The pairing between MVs and CVs is now as follows:

1. $u\left[0, t_{1}\right)$ is implemented open loop as $u\left[0, t_{1}\right)=u_{\max }$.

2. $t_{1}$ is determined when $T_{j}(t)$ reaches $T_{j, \min }$.

3. $u\left[t_{1}, t_{2}\right)$ is determined by tracking the path constraint $T_{j}(t)=T_{j, \min }$.

4. $t_{2}$ is determined from the terminal condition $n_{D}\left(t_{f}\right)=n_{D, \max }$.

5. $\pi$ is determined from the terminal condition $\frac{\partial n_{C}\left(t_{f}\right)}{\partial \pi}=0$.

Steps 1-3 are the same as with Solution model A and are therefore straightforward to implement. Now, both Steps 4 and 5 require prediction, which requires a model to be done online or, otherwise, could be implemented on a run-to-run basis. Steps 4 and 5 represent a $2 \times 2$ control problem. Note that it is no longer necessary to compute the Hamiltonian function $H(t)$.

Solution model C using a reference trajectory to meet the terminal constraint.

This solution model attempts to meet the terminal constraint $n_{D}\left(t_{f}\right)=n_{D, \text { max }}$ online within a single run. 
For this, we define the profile $n_{D, t e r m}(t)$ in the time interval $\left[t_{2}, t_{f}\right]$ that has the property to end up at $n_{D, \max }$ at final time. One could, for example, define a profile that increases linearly between $n_{D, 2}$ at $t_{2}$ and $n_{D, \max }$ at $t_{f}$ :

$$
n_{D, t e r m}(t)=n_{D, 2}+\frac{n_{D, \max }-n_{D, 2}}{t_{f}-t_{2}}\left(t-t_{2}\right) .
$$

This way, the corresponding NCO include three path constraints and three pointwise constraints at times $t_{1}$ and $t_{2}$ and $t_{f}$ (Table 8).

Table 8: NCO for Solution model C (pointwise constraints are underlined).

\begin{tabular}{|c|c|c|}
\hline & Path & Terminal \\
\hline \multirow[t]{5}{*}{ Constraints } & $u\left[0, t_{1}\right)=u_{\max }$ & \multirow[t]{5}{*}{-} \\
\hline & $\underline{T_{j}\left(t_{1}\right)=T_{j, \min }}$ & \\
\hline & $T_{j}\left[t_{1}, t_{2}\right)=T_{j, \min }$ & \\
\hline & $\underline{n_{D}\left(t_{2}\right)=n_{D, 2}}$ & \\
\hline & $n_{D}\left[t_{2}, t_{f}\right]=n_{D, \text { term }}\left[t_{2}, t_{f}\right]$ & \\
\hline Sensitivities & - & $\frac{\partial n_{C}\left(t_{f}\right)}{\partial n_{D, 2}}=0$ \\
\hline
\end{tabular}

The pairing between MVs and CVs is now as follows:

1. $u\left[0, t_{1}\right)$ is implemented open loop as $u\left[0, t_{1}\right)=u_{\max }$.

2. $t_{1}$ is determined when $T_{j}(t)$ reaches $T_{j, \min }$.

3. $u\left[t_{1}, t_{2}\right)$ is determined by tracking the path constraint $T_{j}(t)=T_{j, \min }$.

4. $t_{2}$ is determined when $n_{D}(t)$ reaches the predefined value $n_{D, 2}$ for the current batch.

5. $u\left[t_{2}, t_{f}\right]$ is determined by tracking the path constraint $n_{D}(t)=n_{D, t e r m}(t)$.

Again, Steps 1-3 are the same as with Solution models A and B. What is new is that Steps 4 and 5 are now also straightforward to implement online, provided the concentration of the Species D can be measured or estimated online. 
A comparison of Tables 6-8 calls for the following comments:

- Solution model A, which is able to generate the numerical solution obtained from the plant model, is very impractical for implementation using feedback control. However, note that this parameterization might be rather handy for numerical optimization, for example as an alternative to CVP.

- Solution model B can be implemented via a combination of online and run-to-run control since it does not contain path sensitivities that typically require a model for computation.

- Solution model C contains mostly path constraints that can be handled via online control. The terminal sensitivity $\frac{\partial n_{C}\left(t_{f}\right)}{\partial n_{D, 2}}=0$ is a way of determining the optimal value of $n_{D, 2}$. However, for any practical purpose, the value of $n_{D, 2}$ can be chosen conservatively (slightly smaller than $n_{D, \max }$ ) with negligible effect on the cost.

- All the corresponding tracking schemes attempt to meet the active constraints and push certain gradients to zero. Like with any control scheme, one cannot guarantee, in the presence of disturbances, that the setpoints are met at all times. However, the presence of feedback helps reduce the sensitivity to these disturbances. One finds here all the pros and cons of multivariable feedback control!

\subsubsection{Simulation results}

Optimization results using NCO tracking with the there aforementioned solution models are presented next. It is assumed that uncertainty is present in the form of time-varying kinetic coefficients for the plant:

$$
k_{1}(t)=k_{1,0} \frac{\alpha}{\alpha+t}, \quad k_{2}(t)=k_{2,0} \frac{\beta}{\alpha-t},
$$

with the nominal model values $k_{1,0}=0.11 \mathrm{~L} /(\mathrm{mol} \min ), k_{2,0}=0.13 \mathrm{~L} /(\mathrm{mol} \min ), \alpha=1800 \mathrm{~min}$ and $\beta=900 \mathrm{~min}$, and where $t$ is the time in minutes. These variations might correspond to a change in catalyst activity with time. The information regarding these variations is, of course, not revealed to the optimization algorithms. If this information were available, the ideal cost value would be $J^{*}=392.3 \mathrm{~mol}$ of the desired product $\mathrm{C}$. 
The input profiles with NCO tracking using Solution models A, B and C are shown in Figure $20 .^{\S}$ Though the third arc is quite different in the three cases, the optimal costs are nearly the same, as will be documented later.

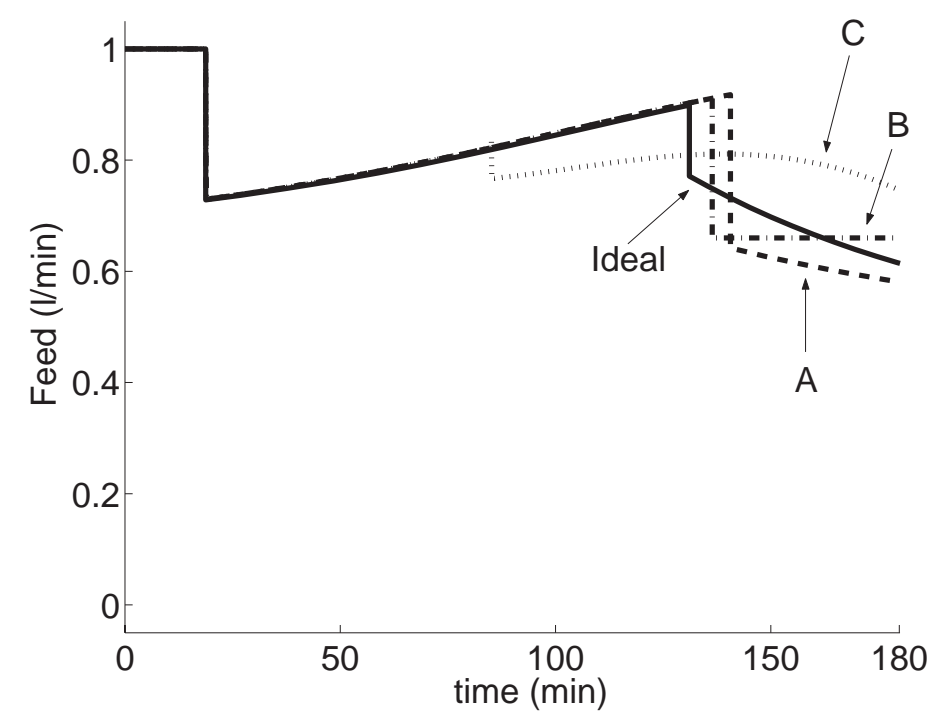

Figure 20: Feedrate profile: Ideal profile computed with complete knowledge of the uncertainty (solid) and after the 5th run with NCO-tracking control based on the three solution models A, B and C.

Optimality measure. With several solution model candidates, it is important to be able to assess the quality of the approximation. For this, the measure $\Theta$ that expresses the ratio of two cost differences is introduced ${ }^{90}$ :

$$
\Theta=\frac{J\left(u^{\text {cons }}\right)-J\left(u^{N C O}\right)}{J\left(u^{c o n s}\right)-J\left(u^{*}\right)},
$$

where $u^{N C O}$ represents the inputs obtained via NCO tracking, $u^{*}$ the true optimal solution, and $u^{\text {cons }}$ a conservative solution used in practice. The true optimal solution $u^{*}$ is typically unknown. However, note that the measure $\Theta$ does not require the optimal inputs $u^{*}$ themselves, but rather the optimal cost $J\left(u^{*}\right)$, which could possibly be obtained by extrapolation of the costs obtained with models of increasing complexity. The optimality measure defined above runs between 0 and 1 and provides the fraction of potential improvement that has been realized. The closer $\Theta$ is to 1 , the better the optimality.

\footnotetext{
$\S$ Solution model A requires a process model to enforce the path condition $H_{u}\left[t_{2}, t_{f}\right]=0$. In this simulation study, since the process model is available, it is possible to "implement" NCO tracking based on Solution model A. In practice, however, NCO tracking works exclusively on the basis of online and run-to-run feedback control, that is, without the need of a process model.
} 
Quantitative comparaison. The results are compared in Table 9, where, for all cases, convergence is achieved in less than 10 runs. For the sake of comparison, application of the optimal input calculated offline using the nominal plant model, $u^{\text {cons }}$, gives the cost $J\left(u^{\text {cons }}\right)=374.6$ mol. The main observations are as follows:

- The constraint on the jacket temperature is active in all cases since it is handled online. The first run with Controllers A and B indicates how much can be gained by meeting that path constraint alone.

- Enforcing the path sensitivity in Controller A using a model and neighboring-extremal control ${ }^{75}$ is not $^{2}$ really better (here even slightly worse) than a crude approximation by a constant value as implemented by Controller B.

- Meeting the terminal constraint improves the cost significantly. This is done via run-to-run control for Controllers A and B and online via tracking of $n_{D, t e r m}(t)$ in Controller C. Hence, with Controller $\mathrm{C}$, all the constraints are met online and thus the cost is nearly optimal from the first run on. The parameter $n_{D, 2}$, which indirectly selects the switching time $t_{2}$, has a negligible effect on the cost and therefore is not adapted in this study. " Hence, all the adjustments with Controller C are made online.

\subsection{Industrial Batch Polymerization}

The last case study illustrates the use of NCO tracking for the optimization of an industrial reactor for the copolymerization of acrylamide ${ }^{91}$. As the polymer is repeatedly produced in a batch reactor, run-to-run NCO tracking using run-end measurements is applied.

\subsubsection{A brief description of the process}

The 1-ton industrial reactor investigated in this section is dedicated to the inverse-emulsion copolymerization of acrylamide and quaternary ammonium cationic monomers, a heterogeneous water-in-oil polymerization

In this example, the terminal sensitivities are so low that there is no need for adaptation. This is valid for $\pi$ in Controller B and $n_{D, 2}$ in Controller C. 


\begin{tabular}{|c|c|c|c|c|c|}
\hline Controller & $\begin{array}{l}\text { Run } \\
\text { index }\end{array}$ & $\begin{array}{l}\text { Min. jacket } \\
\text { temperature } \\
T_{j, \min }=10^{\circ} \mathrm{C}\end{array}$ & $\begin{array}{c}\text { Final amount } \\
\text { of } D(\mathbf{m o l}) \\
n_{D, \text { max }}=100 \mathrm{~mol}\end{array}$ & $\begin{array}{c}\text { Final amount } \\
\text { of } \mathbf{C}(\mathbf{m o l}) \\
\text { Cost } J\end{array}$ & $\begin{array}{c}\text { Optimality } \\
\text { measure } \\
\Theta\end{array}$ \\
\hline Optimal & & 10 & 100 & 392.3 & 1 \\
\hline Open loop & & 10.1 & 72.5 & 374.6 & 0 \\
\hline A & $\begin{array}{c}1 \\
5 \\
10\end{array}$ & $\begin{array}{l}10.0 \\
10.0 \\
10.0\end{array}$ & $\begin{array}{l}79.6 \\
96.2 \\
99.6\end{array}$ & $\begin{array}{l}376.8 \\
389.8 \\
392.1\end{array}$ & $\begin{array}{l}0.12 \\
0.86 \\
0.99\end{array}$ \\
\hline B & $\begin{array}{c}1 \\
5 \\
10\end{array}$ & $\begin{array}{l}10.0 \\
10.0 \\
10.0\end{array}$ & $\begin{array}{l}83.6 \\
98.1 \\
99.9\end{array}$ & $\begin{array}{l}383.3 \\
391.6 \\
392.2\end{array}$ & $\begin{array}{l}0.49 \\
0.96 \\
0.99\end{array}$ \\
\hline $\mathrm{C}$ & $\begin{array}{c}1 \\
5 \\
10\end{array}$ & $\begin{array}{l}10.0 \\
10.0 \\
10.0\end{array}$ & $\begin{array}{l}100 \\
100 \\
100\end{array}$ & $\begin{array}{l}391.8 \\
391.8 \\
391.8\end{array}$ & $\begin{array}{l}0.97 \\
0.97 \\
0.97\end{array}$ \\
\hline
\end{tabular}

Table 9: Comparison of NCO-tracking scenarios for three different controllers and various numbers of runs: Distance to the two constraints, cost $J$ and optimality measure $\Theta$. "Optimal" is with respect to the perturbed system. "Open loop" means open-loop application of the optimal input computed for the nominal (unperturbed) system. Note that open-loop application of the nominal optimal input does not meet the two constraints and is therefore widely suboptimal. 
process. Nucleation and polymerization are confined to the aqueous monomer droplets, while the polymerization follows a free-radical mechanism. Table 10 summarizes the reactions that are known to occur.

Table 10: Main reactions in the inverse-emulsion process.

\section{Oil-phase reactions}

- initiation by initiator decomposition

- reactions of primary radicals

- propagation reactions

\section{Transfer between phases}

- initiator

- co-monomers

- primary radicals

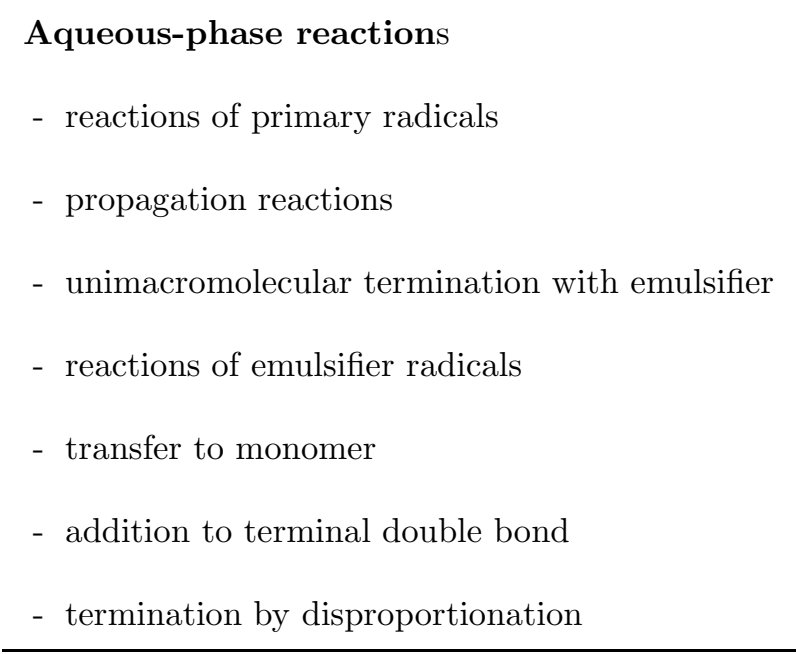

A tendency model capable of predicting the conversion and the average molecular weight was developed. The $7^{\text {th }}$-order model includes balance equations for the two monomers and for the chain-transfer agent, dynamic equations for the zeroth-, first- and second-order moments of the molecular weight distribution and for the efficiency of the initiator. The model parameters have been fitted to match observed data. Note that certain effect are nearly impossible to model. For instance, the efficiency of the initiator can vary significantly between batches because of residual oxygen concentration at the outset of the reaction. Chaintransfer agents and reticulants are also added to help control the molecular weight distribution. These small 
variations in the recipe are not incorporated in the tendency model. Hence, optimization of this process clearly calls for the use of measurement-based techniques.

\subsubsection{Nominal optimization of the tendency model}

The objective is to minimize the reaction time, while meeting four constraints, namely, (i) the terminal molecular weight $X\left(t_{f}\right)$ has to exceed the target value $X_{\min }$ to ensure total conversion of acrylamide, (ii) the terminal conversion $\bar{M}_{w}\left(t_{f}\right)$ has to exceed a target value to ensure total conversion of acrylamide, (iii) heat removal is limited, which is incorporated in the optimization problem by the lower bound $T_{j, i n, \text { min }}$ on the jacket inlet temperature $T_{j, i n}(t)$, and (iv) the reactor temperature $T_{r}(t)$ is upper bounded. The MVs are the reactor temperature profile $T_{r}\left[0, t_{f}\right]$ and the reaction time $t_{f}$. The dynamic optimization problem can be formulated as follows:

$$
\begin{array}{ll}
\min _{T_{r}\left[0, t_{f}\right], t_{f}} & J:=t_{f} \\
\text { s.t. } & \text { dynamic reactor model with initial conditions } \\
& X\left(t_{f}\right) \geq X_{\text {min }}, \\
& \bar{M}_{w}\left(t_{f}\right) \geq \bar{M}_{w, \min }, \\
& T_{j, i n}(t) \geq T_{j, \text { in }, \min }, \\
& T_{r}(t) \leq T_{r, \max } .
\end{array}
$$

\subsubsection{Solution model}

The results of nominal optimization are shown in Figure 21, with normalized values of the reactor temperature $T_{r}(t)$ and of the time $t$. The nominal optimal solution consists of two arcs with the following interpretation:

- Heat-removal limitation. Up to a certain level of conversion, the temperature is limited by heatremoval. Initially, the operation is isothermal and corresponds closely to what is used in industrial practice. Also, this first isothermal arc ensures that the terminal constraint on molecular weight will 


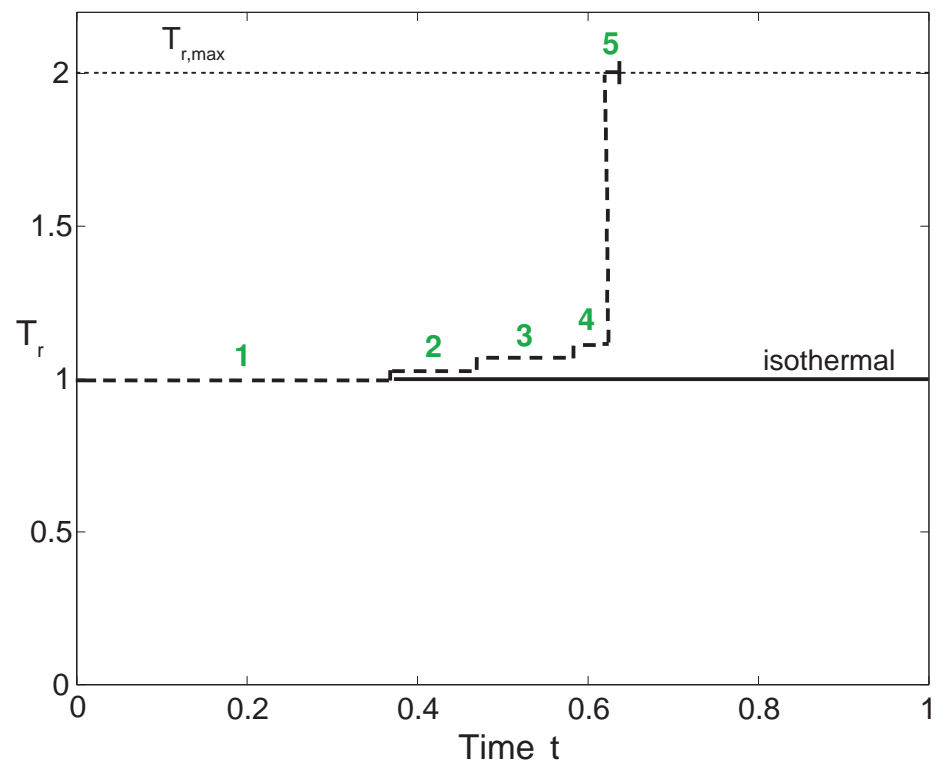

Figure 21: Optimal reactor temperature calculated from the nominal model using 5 piecewise-constant temperature elements of variable duration.

be satisfied as it is mostly determined by the concentration of chain-transfer agent.

- Intrinsic compromise. The second arc represents a compromise between reaction speed and quality.

The decrease in reaction rate due to smaller monomer concentrations is compensated by an increase in temperature, which accelerates the reaction but decreases molecular weight.

This interpretation of the nominal solution is the basis for the solution model. Since operators are reluctant to change the temperature policy during the first part of the batch and the reaction is highly exothermic, it has been decided to:

- Implement the first arc isothermally, with the temperature kept at the value used in industrial practice.

- Implement the second arc adiabatically, that is, without jacket cooling. The reaction mixture is heated up by the reaction, which allows linking the maximal reachable temperature to the amount of reactants (and thus the conversion) at the time of switching. 
With this so-called semi-adiabatic temperature profile, there are only two degrees of freedom, namely, the switching time between the two arcs $t_{s w}$ and the final time $t_{f}$. The dynamic optimization problem can be rewritten as the following static problem (see Section 8.2):

$$
\begin{array}{ll}
\min _{t_{s w}, t_{f}} & t_{f} \\
\text { s.t. } & \text { static model (in this study, the plant) } \\
& X\left(t_{f}\right) \geq X_{\min }, \\
& \bar{M}_{w}\left(t_{f}\right) \geq \bar{M}_{w, \min } \\
& T_{r}\left(t_{f}\right) \leq T_{r, \max } .
\end{array}
$$

Such a reformulation is made possible since:

a. The switching time $t_{s w}$ and the final time $t_{f}$ are fixed at the beginning of the batch, while performance and constraints are evaluated at batch end. This way, the dynamics are lumped into the static map (7) $\left\{\pi: t_{s w}, t_{f}\right\} \rightarrow\left\{z: t_{f}, X\left(t_{f}\right), \bar{M}_{w}\left(t_{f}\right), T_{r}\left(t_{f}\right)\right\}$.

b. Maintaining the temperature constant initially at its current practice value ensures that the heatremoval limitation is satisfied. This constraint can thus be removed from the problem formulation.

Because (i) the constraint on the molecular weight is less restrictive than that on the reactor temperature, (ii) the final time is defined upon meeting the desired conversion, and (iii) the terminal constraint on reactor temperature is active at the optimum, the NCO reduce to the following two conditions:

$$
\begin{aligned}
T_{r}\left(t_{f}\right)-T_{r, \max } & =0, \\
\frac{\partial t_{f}}{\partial t_{s w}}+\nu \frac{\partial\left[T_{r}\left(t_{f}\right)-T_{r, \max }\right]}{\partial t_{s w}} & =0,
\end{aligned}
$$

where $\nu$ is the Lagrange multiplier associated with the constraint on final temperature. The first equation determines the switching time, while the second equation can be used for computing $\nu$, which however is not used in this study. 


\subsubsection{Industrial results}

The solution to the original dynamic optimization problem can be approximated by adjusting the switching time so as to meet the terminal constraint on reactor temperature. This can be implemented using a simple integral run-to-run controller as shown in Figure 22.

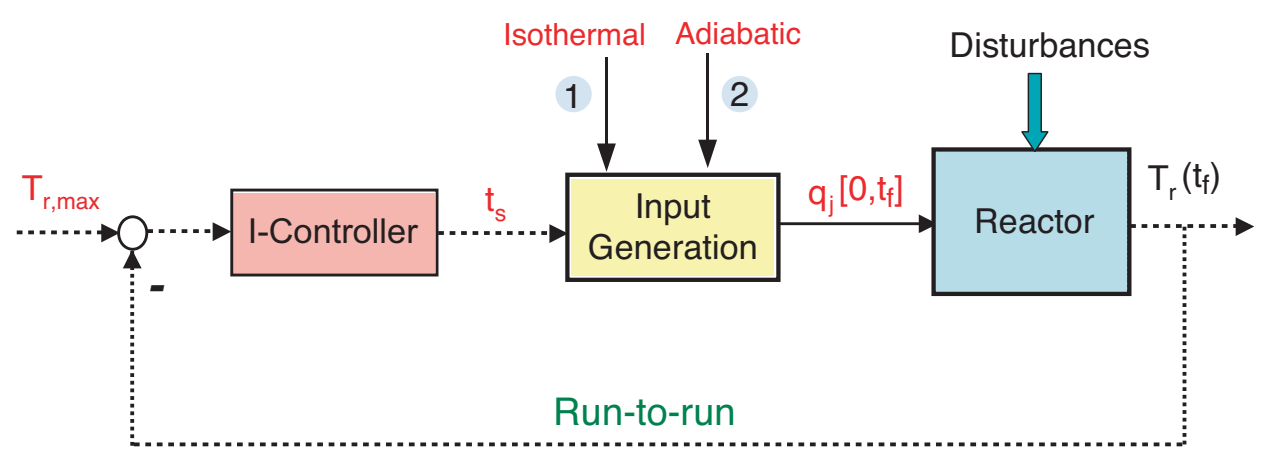

Figure 22: Run-to-run control of the final reactor temperature by adjusting the switching time $t_{s}$. The reactor is initially operated isothermally at the normalized temperature $T_{r, r e f}=1$. Adiabatic operation starts at $t_{s}$, which is adjusted on a run-to-run basis to achieve $T_{r}\left(t_{f}\right)=T_{r, \max }$. The manipulated input is the flowrate of cooling medium in the jacket $q_{j}(t)$, which is generated by a feedback control law to regulate $T_{r}(t)$ around $T_{r, r e f}=1$ in the isothermal phase for $t<t_{s}$; then, $q_{j}\left[t_{s}, t_{f}\right]=0$ is set in the adiabatic phase.

Figure 23 depicts the application of the method to the optimization of the 1-ton industrial reactor. The first batch is performed using a conservative value of the switching time. The reaction time is significantly reduced after only two batches, without any off-spec product. Table 11 summarizes the adaptation results, highlighting the $35 \%$ reduction in reaction time compared to the isothermal policy used in industrial practice. Results could have been even more impressive, but a backoff from the constraint on the final temperature was added for safety purposes. This semi-adiabatic policy has become standard practice for our industrial partner. The same policy has also been implemented, together with the adaptation scheme, to other polymer grades and to larger reactors. 


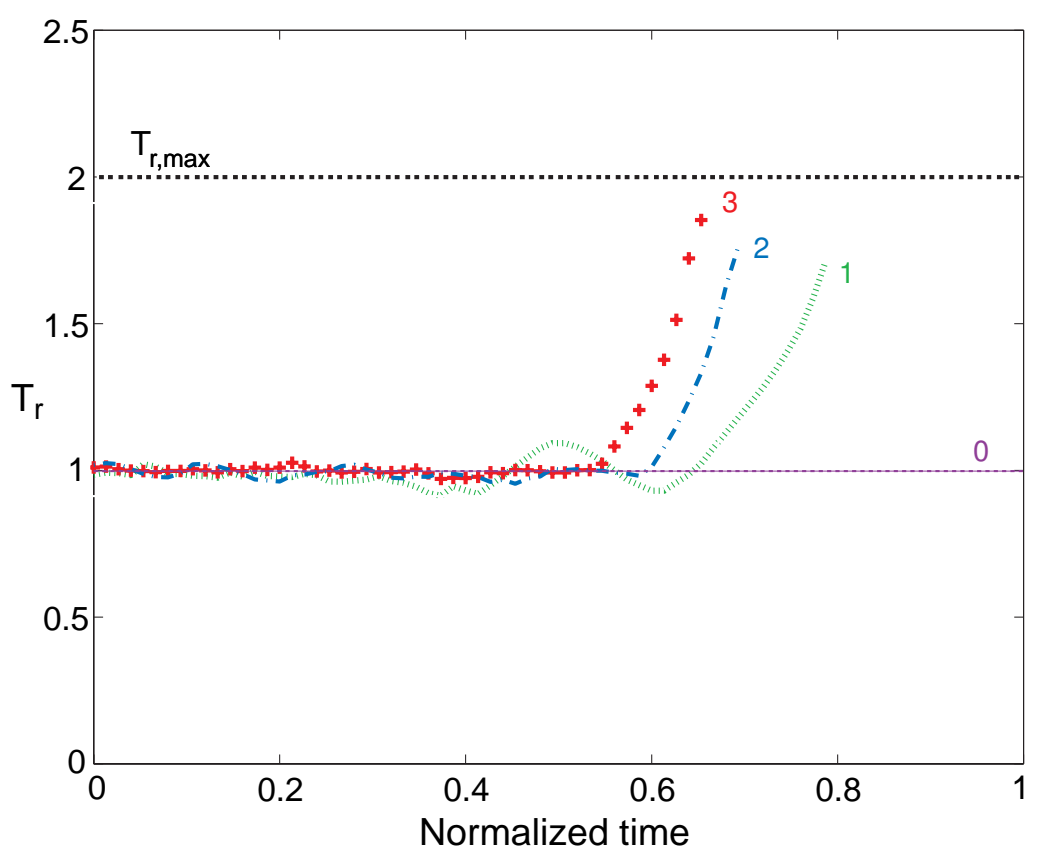

Figure 23: Normalized temperature profiles measured in an industrial reactor as a result of reducing the switching time between isothermal and adiabatic operations. Compared to the normalized reaction time of 1 for the current-practice isothermal operation, the reaction time is successively reduced to $0.78,0.72$, and 0.65 in 3 batches. Note that a backoff from $T_{r, \max }$ is implemented for safety purposes, mainly to account for run-time disturbances that cannot be handled by this technique.

Table 11: Run-to-run optimization results for a 1-ton copolymerization reactor.

\begin{tabular}{ccccc}
\hline \hline Batch & Strategy & $t_{s w}$ & $T_{r}\left(t_{f}\right)$ & $t_{f}$ \\
\hline 0 & Isothermal & & 1.00 & 1.00 \\
\hline 1 & Semi-adiabatic & 0.65 & 1.70 & 0.78 \\
\hline 2 & Semi-adiabatic & 0.58 & 1.78 & 0.72 \\
\hline 3 & Semi-adiabatic & 0.53 & 1.85 & 0.65 \\
\hline
\end{tabular}




\section{Conclusions}

\subsection{Summary}

This chapter has analyzed the operation of batch and semi-batch processes with respect to both control and optimization. There often is a great potential for process improvement in batch processes. Although the major gains are concerned with chemical choices regarding the reaction and separation steps, process operation also carries a significant potential for cost reduction. However, cost savings in process operations are often associated with automation rather than advanced control. The former term encompasses the hardware and software elements needed to operate an industrial process as automatically as possible. These include automatic start-up and shut-down operations, process monitoring, fault diagnosis, alarm handling, automatic sampling and analysis. The latter term is concerned mainly with algorithmic solutions for solving well-defined problems and, as such, might only represent $10 \%$ of the control and automation activities in the batch process industry.

This chapter has also shown that incorporating measurements in the optimization framework helps improve the performances of chemical processes despite the use of models of limited accuracy. The various RTO methods differ in the way measurements are used and the inputs are adjusted to reject the effect of uncertainty. Measurements can be utilized to iteratively (i) update the states or the parameters of the model that is used for optimization, or (ii) adjust the inputs via feedback control to enforce the NCO. It has been argued that the latter technique has the ability of rejecting the effect of uncertainty in the form of plant-model mismatch and process disturbances.

\subsection{Future Challenges}

While several of the techniques presented here have been implemented successfully in industrial practice, many challenging problems still remain. Here are a few of them: 
- Advances in optimization techniques in general, and in model predictive control in particular, have undoubtedly influenced industrial implementation. However, these techniques depend on the availability of process models of reasonably high fidelity and modest complexity, which is difficult to achieve in practice. Methods of nonlinear model reduction capable of representing complex process dynamics effectively with reduced-order, control-relevant models will provide a significant boost to the industrial application of advanced control and optimization techniques.

- While advances in state estimation have enabled the estimation of infrequently measured product characteristics, these estimates can never completely replace the actual measurements themselves. And as the manufacturing chain becomes inexorably more tightly integrated, each successive downstream customer will place increasingly stringent performance demands on the products they receive from each of their suppliers. Meeting these demands will ultimately require process control performance levels that cannot be attained without better measurement or estimation of product properties. Advances in sensors, analyzers and ancillary measurement technology will be required in order to make measurements of product properties more frequently available than is currently possible.

- As the structure of process control systems gets more complex, analyses of model dynamics, overall system stability and achievable performance will be essential, especially for providing guidance in selecting the best alternative for each problem.

\section{Acknowledegments}

The authors would like to thank the former and present group members at EPFL's Laboratoire d'Automatique, who contributed many of the insights and results presented here. A particular thank goes to Dr. Alejandro Marchetti and Dr. René Schneider who provided useful feedback on the manuscript. 


\section{References}

1. D. Bonvin. Control and optimization of batch processes. In J. Baillieul and T. Samad, editors, Encyclopedia of Systems and Control, pages 1-6. Springer, 2014.

2. D. Bonvin. Optimal operation of batch reactors - A personal view. J. Process Contr., 8(5-6):355-368, 1998.

3. U. Diwekar. Batch Processing: Modeling and Design. CRC Press, 2014.

4. K. Y. Rani. Optimization and Control of Semi-Batch Reactors: Data-Driven Model-Based Approaches. LAP Lambert Academic Publishing, 2011.

5. F. Caccavale, M. Iamarino, F. Pierri, and V. Tufano. Control and Monitoring of Chemical Batch Reactors. Advances in Industrial Control. Springer-Verlag, New York, 2011.

6. I. M. Mujtaba. Batch Distillation: Design and Operation. Series on Chemical Engineering: Vol. 3. Imperial College Press, London UK, 2004.

7. U. Diwekar. Batch Distillation: Simulation, Optimal Design, and Control. Series in Chemical and Mechanical Engineering. CRC Press, 1995.

8. H. Seki, N. Furuya, and S. Hoshino. Evaluation of controlled cooling for seeded batch crystallization incorporating dissolution. Chem. Eng. Sci., 77(10):10-17, 2012.

9. D. Wieckhusen. Development of batch crystallizations. In W. Beckmann, editor, Crystallization: Basic Concepts and Industrial Applications, pages 187-202. Wiley-VCH, 2013.

10. L. Ljung. System Identification: Theory for the User. Prentice Hall, 1999.

11. R. K. Pearson and B. A. Ogunnaike. Nonlinear Process Identification. Prentice-Hall, Inc., 1996.

12. D. M. Himmelblau. Applications of artificial neural networks in chemical engineering. Korean Journal of Chemical Engineering, 17(4):373-392, 2000.

13. S. A. Russel, P. Kesavan, J. H. Lee, and B. A. Ogunnaike. Recursive data-based prediction and control of batch product quality. AIChE J., 44(11):2442-2458, 1998. 
14. W. G. Cochran and G. M. Cox. Experimental Designs. John Wiley, 1992.

15. B. Srinivasan and D. Bonvin. Controllability and stability of repetitive batch processes. J. Process Contr., 17:285-295, 2007.

16. C. Pertev, M. Turker, and R. Berber. Dynamic modelling, sensitivity analysis and parameter estimation of industrial yeast fermenters. Comp. Chem. Eng., 21:S739-S744, 1997.

17. D. C. Montgomery. Design and Analysis of Experiments. John Wiley \& Sons, New York, 8th edition, 2013.

18. S. B. Jorgensen and K. M. Hangos. Grey-box modelling for control: Qualitative models as unifying framework. Int. J. Adaptive Control and Signal Proc., 9(6):547-562, 1995.

19. H. J. A. F. Tulleken. Application of the grey-box approach to parameter estimation in physico-chemical models. In IEEE CDC, pages 1177-1183, Brighton, UK, 1991.

20. C. Filippi, J. L. Greffe, J. Bordet, J. Villermaux, J. L. Barnay, B. Ponte, and C. Georgakis. Tendency modeling of semi-batch reactors for optimization and control. Comp. Chem. Eng., 41:913-920, 1986.

21. G. François, B. Srinivasan, and D. Bonvin. Use of measurements for enforcing the necessary conditions of optimality in the presence of constraints and uncertainty. J. Process Contr., 15(6):701-712, 2005.

22. B. A. Ogunnaike and W. H. Ray. Process Dynamics, Modeling and Control. Oxford University Press, 1994.

23. D. E. Seborg, T. F. Edgar, D. A. Mellichamp, and F. J. Doyle. Process Dynamics and Control. John Wiley, New York, 2004.

24. K. J. Aström and B. Wittenmark. Adaptive Control. Addison-Wesley, Reading, Massachusetts, 2nd edition, 1995.

25. P. Apkarian and R. Adams. Advanced gain-scheduling techniques for uncertain systems. IEEE Trans. Control System Tech., 6:21-32, 1998. 
26. V. Hagenmeyer and M. Nohr. Flatness-based two-degree-of-freedom control of industrial semi-batch reactors using a new observation model for an extended Kalman filter approach. Int. J. of Control, 81(3):428-438, 2008.

27. M. Shahrokhi and M. Ali Fanaei. Nonlinear temperature control of a batch suspension polymerization reactor. Polymer Engineering and Science, 42(6):1296-1308, 2002.

28. M. Soroush and C. Kravaris. Nonlinear control of a batch polymerization reactor: An experimental study. AIChE J., 38(9):1429-1448, 1992.

29. M. R. Juba and J. W. Hamer. Progress and challenges in batch process control. In Chemical Process Control - CPC-III, pages 139-183, Asilomar, CA, 1986.

30. R. A. Wright and C. Kravaris. Nonminimum-phase compensation for nonlinear processes. AIChE J., $38(1): 2640,1992$.

31. Mettler-Toledo. OptiMax Heat-Flow Calorimetry. http://ch.mt.com, 2016.

32. B. Ogunnaike, G. François, M. Soroush, and D. Bonvin. Control of polymerization processes. In W. S. Levine, editor, The Control Handbook - Control System Applications, pages 12.1-12.23. CRC Press, 2011.

33. J. B. Rawlings and D. Q. Mayne. Model Predictive Control: Theory and Design. Nob Hill Pub, 2009.

34. Z. K. Nagy and R. D. Braatz. Robust nonlinear model predictive control of batch processes. AIChE J., 49(7):1776-1786, 2003.

35. R. G. Brown. Introduction to Random Signal Analysis and Kalman Filtering. John Wiley \& Sons, New York, 1983 .

36. E. W. Kamen and J. K. Su. Introduction to Optimal Estimation. Advanced Textbooks in Control and Signal Processing. Springer Verlag, 1999.

37. J. Valappil and C. Georgakis. Nonlinear model predictive control of end-use properties in batch reactors. AIChE J., 48(9):2006 - 2021, 2002. 
38. J. Flores-Cerrillo and J. F. MacGregor. Within-batch and batch-to-batch inferential-adaptive control of semibatch reactors: A partial least squares approach. Ind. Eng. Chem. Res., 42:3334-3335, 2003.

39. Y. Yabuki and J. F. MacGregor. Product quality control in semi-batch reactors using mid-course correction policies. Ind. Eng. Chem. Res., 36:1268-1275, 1997.

40. J. Flores-Cerrillo and J. F. MacGregor. Control of batch product quality by trajectory manipulation using latent variable models. J. Process Contr., 14:539-553, 2004.

41. C. Welz, B. Srinivasan, and D. Bonvin. Measurement-based optimization of batch processes: Meeting terminal constraints on-line via trajectory following. J. Process Contr., 18(3-4):375-382, 2008.

42. K. L. Moore. Iterative Learning Control for Deterministic Systems. Springer-Verlag, Advances in Industrial Control, London, 1993.

43. S. Arimoto, S. Kawamura, and F. Miyazaki. Bettering operation of robots by learning. J. Robotic Syst., $1(2): 123-140,1984$.

44. J. Wallen, S. Gunnarsson, R. Henriksson, S. Moberg, and M. Norrlof. ILC applied to a flexible two-link robot model using sensor-fusion-based estimates. In IEEE CDC, pages 458-463, Shanghai, China, 2009.

45. J. H. Lee and K. S. Lee. Iterative learning control applied to batch processes: An overview. Control Eng. Practice, 15(10):1306-1318, 2007.

46. Y. Wang, F. Gao, and F. J. Doyle. Survey on iterative learning control, repetitive control, and run-to-run control. J. of Process Contr., 19(10):1589-1600, 2009.

47. S. Arimoto, T. Naniwa, and H. Suzuki. Robustness of P-type learning control with a forgetting factor for robotic motion. In 29th Conference on Decision and Control, pages 2640-2645, Honolulu, Hawaii, December 1990 .

48. G. Heinzinger, D. Fenwick, B. Paden, and F. Miyazaki. Stability of learning control with disturbances and uncertain initial conditions. IEEE Trans. Autom. Contr., 37:110-114, 1992. 
49. C. Welz, B. Srinivasan, and D. Bonvin. Iterative learning control with input shift. In IFAC DYCOPS'7, Cambridge, MA, 2004.

50. G. François, B. Srinivasan, and D. Bonvin. A globally convergent algorithm for the run-to-run control of systems with sector nonlinearities. Ind. Eng. Chem. Res., 50(3):1410-1418, 2011.

51. J.-J. E. Slotine and W. Li. Applied Nonlinear Control. Prentice-Hall, 1991.

52. W. C. Thompson. Batch process control. In G. K. Mc Millan and D. M. Considine, editors, Process/Industrial Instruments and Control Handbook, pages 3.70-3.84. MacGraw-Hill, 1999.

53. T. Hudak. Programmable controllers. In G. K. Mc Millan and D. M. Considine, editors, Process/Industrial Instruments and Control Handbook, pages 3.32-3.50. MacGraw-Hill, 1999.

54. R. Netto and A. Bagri. Programmable logic controllers. Int. J. of Computer Applications, 77(11):27-31, 2013.

55. A. G. Marchetti. Modifier-Adaptation Methodology for Real-time Optimization. Doctoral thesis No. 4449, EPFL Lausanne, Switzerland, 2009.

56. B. Galloway and G. P. Hancke. Introduction to industrial control networks. IEEE Comm. Surveys E Tutorials, 15(2):860-880, 2013.

57. D. Ruppen, D. Bonvin, and D. W. T. Rippin. Implementation of adaptive optimal operation for a semi-batch reaction system. Comp. Chem. Eng., 22:185-189, 1998.

58. W. Bequette. Process Dynamics: Modeling, Analysis and Simulation. Prentice Hall, 1998.

59. I. Eckerman. THE BHOPAL SAGA - Causes and Consequences of the World's Largest Industrial Disaster. University Press (India) Private Limited, 2005.

60. A. Marchetti, M. Amrhein, B. Chachuat, and D. Bonvin. Scale-up of batch processes via decentralized control. In IFAC ADCHEM'06, Gramado, Brazil, 2006.

61. C. Welz, B. Srinivasan, and D. Bonvin. Combined online and run-to-run optimization of batch processes with terminal constraints. In IFAC ADCHEM'04, Hong-Kong, 2004. 
62. G. Stephanopoulos. Chemical Process Control: An Introduction to Theory and Practice. Prentice-Hall, 1984.

63. G. François and D. Bonvin. Measurement-based real-time optimization of chemical processes. In S. Pushpavanam, editor, Control and Optimisation of Process Systems, volume 44 of Advances in Chemical Engineering. Elsevier, 2013.

64. O. Rotava and A. C. Zanin. Multivariable control and real-time optimization - An industrial practical view. Hydrocarbon Process., 84(6):61-71, 2005.

65. S. Boyd and L. Vandenberghe. Convex Optimization. Cambridge University Press, 2004.

66. T. E. Marlin and A. N. Hrymak. Real-time operations optimization of continuous processes. In AIChE Symposium Series - CPC-V, volume 93, pages 156-164, 1997.

67. Y. Zhang, D. Monder, and J. F. Forbes. Real-time optimization under parametric uncertainty: A probability constrained approach. J. Process Contr., 12:373-389, 2002.

68. J. F. Forbes, T. E. Marlin, and J. F. MacGregor. Model adequacy requirements for optimizing plant operations. Comp. Chem. Eng., 18(6):497-510, 1994.

69. A. Marchetti, B. Chachuat, and D. Bonvin. Modifier-adaptation methodology for real-time optimization. Ind. Eng. Chem. Res., 48(13):6022-6033, 2009.

70. S. Skogestad. Self-optimizing control: The missing link between steady-state optimization and control. Comp. Chem. Eng., 24:569-575, 2000.

71. K. B. Ariyur and M. Krstic. Real-Time Optimization by Extremum-Seeking Control. John Wiley, New York, 2003.

72. B. Srinivasan and D. Bonvin. Real-time optimization of batch processes via tracking the necessary conditions of optimality. Ind. Eng. Chem. Res., 46(2):492-504, 2007.

73. B. Srinivasan, S. Palanki, and D. Bonvin. Dynamic optimization of batch processes: I. Characterization of the nominal solution. Comp. Chem. Eng., 44:1-26, 2003. 
74. L. T. Biegler. Nonlinear Programming: Concepts, Algorithms, and Applications to Chemical Processes. MOS-SIAM Series on Optimization, 2010.

75. A. E. Bryson and Y. C. Ho. Applied Optimal Control. Hemisphere, Washington DC, 1975.

76. V. S. Vassiliadis, R. W. H. Sargent, and C. C. Pantelides. Solution of a class of multistage dynamic optimization problems. 1. Problems without path constraints. Ind. Eng. Chem. Res., 33(9):2111-2122, 1994.

77. J. S. Logsdon and L. T. Biegler. Accurate solution of differential-algebraic optimization problems. Ind. Eng. Chem. Res., 28(11):1628-1639, 1989.

78. A. E. Bryson. Dynamic Optimization. Addison-Wesley, Menlo Park, California, 1999.

79. M. S. Bazarra, H. D. Sherali, and C. M. Shetty. Nonlinear Programming: Theory and Algorithms. John Wiley and Sons, 2nd edition, New York, USA, 1993.

80. P. E. Gill, W. Murray, and M. H. Wright. Practical Optimization. Academic Press, London, 1981.

81. A. Forsgren, P. E. Gill, and M. H. Wright. Interior-point methods for nonlinear optimization. SIAM Rev., 44(4):525-597, 2002.

82. B. Srinivasan, L. T. Biegler, and D. Bonvin. Tracking the necessary conditions of optimality with changing set of active constraints using a barrier-penalty function. Comp. Chem. Eng., 32(3):572-579, 2008.

83. C. K. Chui and G. Chen. Kalman Filtering with Real-Time Applications. Springer Verlag, 4th edition, 2009.

84. J. B. Rawlings. Moving horizon estimation. In J. Baillieul and T. Samad, editors, Encyclopedia of Systems and Control, pages 1-7. Springer, 2014.

85. D. J. Kozub and J. F. MacGregor. State estimation for semi-batch polymerization reactors. Chem. Eng. Sci., 47:1047-1062, 1992. 
86. D. Dochain. State and parameter estimation in chemical and biochemical processes: A tutorial. J. Process Contr., 13(8):801-818, 2003.

87. B. Wittenmark. Adaptive dual control methods: An overview. In IFAC Symp. on Adaptive Syst. in Control and Signal Proc., pages 67-72, Budapest, 1995.

88. D. Angeli. Economic model predictive control. In J. Baillieul and T. Samad, editors, Encyclopedia of Systems and Control, pages 1-9. Springer, 2014.

89. A. G. Marchetti, B. Chachuat, and D. Bonvin. A dual modifier-adaptation approach for real-time optimization. J. Process Contr., 20(9):1027-1037, 2010.

90. C. Welz, A. G. Marchetti, B. Srinivasan, D. Bonvin, and N. L. Ricker. Validation of a solution model for the optimization of a batch distillation column. In American Control Conference, Portland, Oregon, 2005 .

91. G. François, B. Srinivasan, D. Bonvin, J. Hernandez Barajas, and D. Hunkeler. Run-to-run adaptation of a semi-adiabatic policy for the optimization of an industrial batch polymerization process. Ind. Eng. Chem. Res., 43(23):7238-7242, 2004. 